\title{
Prime factors of dynamical sequences
}

\author{
By Xander Faber and Andrew Granville at Montréal
}

\begin{abstract}
Let $\phi(t) \in \mathbb{Q}(t)$ have degree $d \geqq 2$. For a given rational number $x_{0}$, define $x_{n+1}=\phi\left(x_{n}\right)$ for each $n \geqq 0$. If this sequence is not eventually periodic, and if $\phi$ does not lie in one of two explicitly determined affine conjugacy classes of rational functions, then $x_{n+1}-x_{n}$ has a primitive prime factor in its numerator for all sufficiently large $n$. The same result holds for the exceptional maps provided that one looks for primitive prime factors in the denominator of $x_{n+1}-x_{n}$. Hence the result for each rational function $\phi$ of degree at least 2 implies (a new proof) that there are infinitely many primes. The question of primitive prime factors of $x_{n+\Delta}-x_{n}$ is also discussed for $\Delta$ uniformly bounded.
\end{abstract}

\section{Introduction}

For a given sequence of non-zero integers $\left\{x_{n}\right\}_{n \geq 0}$, a primitive prime factor of $x_{n}$ is a prime $p_{n}$ that divides $x_{n}$ but does not divide any term $x_{m}$ with $0 \leqq m<n$. For example, the non-zero terms of the Fibonacci sequence $a_{0}=0, a_{1}=1$ and $a_{n+2}=a_{n+1}+a_{n}$ have a primitive prime factor for every $n>12$ (see [3]).

We call $p_{n}$ a super-primitive prime factor of $x_{n}$ if $p_{n} \nmid x_{m}$ for all $m \neq n$. The Fermat numbers $F_{n}=2^{2^{n}}+1$ are pairwise coprime, and so have a super-primitive prime factor for every $n \geqq 0$.

Given a rational function $\phi(t)$ and a point $x_{0} \in \mathbb{C} \cup\{\infty\}$, we define $x_{n+1}=\phi\left(x_{n}\right)$ for each $n \geqq 0$. We have already seen an example, namely the sequence of Fermat numbers. Indeed, note that

$$
F_{n+1}-2=2^{2^{n+1}}-1=\left(2^{2^{n}}+1\right)\left(2^{2^{n}}-1\right)=F_{n}\left(F_{n}-2\right),
$$

and so if $\phi(t)=t^{2}-2 t+2$ and $F_{0}=3$, then $F_{n+1}=\phi\left(F_{n}\right)$ for each $n \geqq 0$.

Our first goal was to show that, for any $\phi(t) \in \mathbb{Q}(t)$, the numerator of $x_{n+1}-x_{n}$ contains a primitive prime factor for all sufficiently large $n$ provided $\left\{x_{n}\right\}_{n \geqq 0}$ does not

The authors are partially supported by NSERC, and the first author is supported by an NSF postdoctoral fellowship. 
eventually become periodic (which is equivalent to the statement that the $x_{n}$ are distinct). However this is not always true: Let $x_{0}=1$ and $x_{n+1}=x_{n}^{2} /\left(2 x_{n}+1\right)$ for $n \geqq 0$. One verifies by induction that if $F_{n}$ is the $n$th Fermat number, then

$$
x_{n}=\frac{1}{F_{n}-2} \quad \text { and } \quad x_{n+1}-x_{n}=-\frac{2^{2^{n}}}{F_{n+1}-2},
$$

so that 2 is the only prime divisor of the numerator of $x_{n+1}-x_{n}$ for all $n \geqq 0$. Note that the denominator of $x_{n}$ has a primitive prime factor for all $n>0$, which also divides the denominator of $x_{n+1}$. This is what we prove in general: Define $\mathscr{F}_{1}$ to be those $\phi(t) \in \mathbb{Q}(t)$ of the form $\sigma^{-1} \circ \psi \circ \sigma$ for some linear transformation $\sigma(t)=\lambda t+\beta$ with $\lambda \neq 0$, where

$$
\psi(t)=\frac{t^{2}}{t+1} \text { or } \frac{t^{2}}{2 t+1} .
$$

Theorem 1. Suppose that $\phi(t) \in \mathbb{Q}(t)$ has degree $d \geqq 2$, and that a positive integer $\Delta$ is given. Let $x_{0} \in \mathbb{Q}$ and define $x_{n+1}=\phi\left(x_{n}\right)$ for each $n \geqq 0$. If the sequence $\left\{x_{n}\right\}_{n \geqq 0}$ is not eventually periodic, then the numerator of $x_{n+\Delta}-x_{n}$ has a primitive prime factor for all sufficiently large $n$, except if $\phi \in \mathscr{F}_{1}$ and $\Delta=1$. (If $x_{n}$ or $x_{n+\Delta}$ is $\infty$, interpret $x_{n+\Delta}-x_{n}$ as the "fraction" 1/0.) In the case $\phi \in \mathscr{F}_{1}$ and $\Delta=1$, the numerator of $x_{n+1}-x_{n}$ has the same prime factors for all $n$, and for all sufficiently large $n$ the denominator of $x_{n}$ has a primitive prime factor, which also divides the denominator of $x_{n+1}$.

The two affine conjugacy classes of rational functions in $\mathscr{F}_{1}$ are exceptions to the theorem for dynamical reasons. They are characterized by the fact that the point at infinity is fixed with small multiplicity (one or two), and all of the other fixed points are totally ramified. See Lemma 3 and the proof of Theorem 1.

We call $p_{\Delta, n}$ a doubly primitive prime factor if $p_{\Delta, n}$ divides the numerator of $x_{n+\Delta}-x_{n}$, and if $N \geqq n$ and $D \geqq \Delta$ whenever $p_{\Delta, n}$ divides the numerator of $x_{N+D}-x_{N}$. Ingram and Silverman ([5], Conjecture 20) conjectured that the numerator of $x_{n+\Delta}-x_{n}$ has a doubly primitive prime factor for all $\Delta \geqq 1$ and $n \geqq 0$, other than for finitely many exceptional pairs $(\Delta, n)$. Unfortunately their conjecture is false with $\Delta=1$ for any $\phi \in \mathscr{F}_{1}$, though we believe that an appropriate modification is true: Let $\mathscr{B}_{\Delta, d}$ be the set of all $\phi(t) \in \mathbb{C}(t)$ of degree $d$ such that $\phi$ has no periodic point of exact period $\Delta$. A result of Baker (see $\S 7$ and Appendix B) shows that $\mathscr{B}_{\Delta, d}$ is non-empty if and only if $(\Delta, d)$ is one of the pairs $(2,2),(2,3),(2,4)$ or $(3,2)$. Define $\mathscr{F}_{2}$ to be the union of $\mathscr{B}_{2, d}$ for $d=2,3,4$ along with all rational maps $\phi(t)$ of the form $\phi=\sigma^{-1} \circ \psi \circ \sigma$ for some $\sigma(t)=(\alpha t-\beta) /(\gamma t-\delta)$ with $\alpha \delta-\beta \gamma \neq 0$ and $\psi(t)=1 / t^{2}$. Define $\mathscr{F}_{3}=\mathscr{B}_{3,2}$. Corollary 2 in $\S 7$ shows that the classes $\mathscr{F}_{2}$ and $\mathscr{F}_{3}$ provide further counterexamples to the conjecture of Ingram and Silverman, and we believe that should be all of them.

Conjecture. Suppose that $\phi(t) \in \mathbb{Q}(t)$ has degree $d \geqq 2$. Let $x_{0} \in \mathbb{Q}$ and define $x_{n+1}=\phi\left(x_{n}\right)$ for each $n \geqq 0$, and suppose that the sequence $\left\{x_{n}\right\}_{n \geqq 0}$ is not eventually periodic. The numerator of $x_{n+\Delta}-x_{n}$ has a doubly primitive prime factor for all $n \geqq 0$ and $\Delta \geqq 1$, except for those pairs with $\Delta=1,2$ or 3 when $\phi \in \mathscr{F}_{1}, \mathscr{F}_{2}$ or $\mathscr{F}_{3}$, respectively, as well as for finitely many other exceptional pairs $(\Delta, n)$.

In fact we can prove a strengthening of Theorem 1, which implies that if the above conjecture is false, then there must be exceptional pairs $(\Delta, n)$ with $\Delta$ arbitrarily large. 
Theorem 2. Suppose that $\phi(t) \in \mathbb{Q}(t)$ has degree $d \geqq 2$. Let $x_{0} \in \mathbb{Q}$ and define $x_{n+1}=\phi\left(x_{n}\right)$ for each $n \geqq 0$, and suppose that the sequence $\left\{x_{n}\right\}_{n \geq 0}$ is not eventually periodic. For any given $M \geqq 1$, the numerator of $x_{n+\Delta}-x_{n}$ has a doubly primitive prime factor for all $n \geqq 0$ and $M \geqq \Delta \geqq 1$, except for those pairs with $\Delta=1,2$ or 3 when $\phi \in \mathscr{F}_{1}, \mathscr{F}_{2}$ or $\mathscr{F}_{3}$, respectively, as well as for finitely many other exceptional pairs $(\Delta, n)$.

Upon iterating the relation $F_{n+1}-2=F_{n}\left(F_{n}-2\right)$, we see that

$$
F_{n+1}-F_{n}=2^{2^{n+1}}-2^{2^{n}}=2^{2^{n}}\left(F_{n}-2\right)=2^{2^{n}} F_{n-1}\left(F_{n-1}-2\right)=\cdots=2^{2^{n}} F_{n-1} F_{n-2} \cdots F_{1} F_{0} .
$$

Hence $F_{n+1}-F_{n}$ has the same primitive prime factor $p_{n-1}$ as $F_{n-1}$, but there can be no super-primitive prime factor since if $p_{n-1}$ divides $F_{n-1}$, then $p_{n-1}$ divides $F_{N+1}-F_{N}$ for all $N \geqq n$. On the other hand, we saw that all prime factors of $F_{n}$ are super-primitive, and this does generalize as we see in the following result (from which Theorems 1 and 2 are deduced). For this statement, a point $x_{0}$ is called preperiodic if and only if the sequence $\left\{x_{n}\right\}_{n \geqq 0}$ is eventually periodic.

Proposition 1. Let $K$ be a number field. Suppose that $\phi(t) \in K(t)$ has degree $d \geqq 2$, and that 0 is a preperiodic point, but not periodic. If the sequence of $K$-rationals $\left\{x_{n}\right\}_{n \geq 0}$ is not eventually periodic, then the numerator of $x_{n}$ has a super-primitive prime (ideal) factor $P_{n}$ for all sufficiently large $n^{1)}$

Although we discovered Proposition 1 independently, we later learned that it appears as a special case of [5], Theorem 7. Our general strategy is virtually identical to that of [5], but we have simplified the main Diophantine step in the argument. (Our proof avoids the use of Roth's theorem, and instead proceeds by solving a certain Thue/Mahler equation. We discuss this further at the end of the section.)

We will prove a result analogous to Proposition 1 when 0 is a periodic point in Section 4 , though in this case one does not find super-primitive prime factors. Indeed, if 0 has period $q$ and if $P$ divides the numerator of $x_{n}$, then $P$ divides the numerator of $x_{n+k q}$ for all $k \geqq 0$, other than for finitely many exceptional primes $P$.

The proof of Proposition 1 is based on the following sketch of the special case $\phi(t)=t^{2}-2 t+2$ and $x_{0} \in \mathbb{Z}$ (which includes another proof for the special case $x_{0}=F_{0}=3$, so that $x_{n}=F_{n}$ for all $\left.n \geqq 0\right)$. For this $\phi$ we see that 0 is preperiodic but not periodic: $\phi(0)=\phi(2)=2$. Now $x_{n+1}=\phi\left(x_{n}\right) \equiv \phi(0)=2\left(\bmod x_{n}\right)$, and for $m>n$ we then have, by induction, that $x_{m+1}=\phi\left(x_{m}\right) \equiv \phi(2)=2\left(\bmod x_{n}\right)$. Hence if $m>n$, then $\left(x_{m}, x_{n}\right)=\left(2, x_{n}\right)$ which divides 2 , and so any odd prime factor of $x_{n}$ is super-primitive. If $x_{n}$ does not have an odd prime factor, then $x_{n}= \pm 2^{k}$, and there are only finitely many such $n$ as there are only finitely many integers $r$ for which $\phi(r)= \pm 2^{k}$, and no two $x_{n}$ can equal the same value of $r$, else the sequence is eventually periodic.

The deduction of Theorem 1 is based on the following sketch of the special case $\phi(t)=t^{2}+3 t+1$ and $\Delta=1$, and $x_{0} \in \mathbb{Z}$. Here $\phi(-2)=\phi(-1)=-1$, so that -2 is preperiodic but not periodic. To be able to apply Proposition 1, we make a linear change

1) See Section 2 for an explanation of the "numerator" of an element of $K$. 
of variables and consider $\psi(t)=\phi(t-2)+2=t^{2}-t+1$ so that $\psi(0)=\psi(1)=1$; that is, 0 is preperiodic but not periodic. We see that if $y_{0}=x_{0}+2$ and $y_{n+1}=\psi\left(y_{n}\right)$, then $y_{n}=x_{n}+2$ by induction. So Proposition 1 shows $y_{n}=x_{n}+2$ has a super-primitive prime factor $p_{n}$ for all $n$ sufficiently large. But then $p_{n}$ divides $x_{n+2}-x_{n+1}$ since

$$
x_{n+2}=\phi\left(\phi\left(x_{n}\right)\right) \equiv \phi(\phi(-2))=\phi(-1)=\phi(-2) \equiv \phi\left(x_{n}\right)=x_{n+1} \quad\left(\bmod p_{n}\right),
$$

while $p_{n}$ cannot divide $x_{k+1}-x_{k}$ for any $k \leqq n$, else

$$
-1=\phi(-2) \equiv \phi\left(x_{n}\right)=\underbrace{\phi \circ \cdots \circ \phi}_{n-k \text { times }}\left(x_{k+1}\right) \equiv \underbrace{\phi \circ \cdots \circ \phi}_{n-k \text { times }}\left(x_{k}\right)=x_{n} \equiv-2 \quad\left(\bmod p_{n}\right) .
$$

Hence any super-primitive prime factor $p_{n}$ of $y_{n}=x_{n}+2$ is also a primitive prime factor of $x_{n+2}-x_{n+1}$.

Having a super-primitive prime factor is, by definition, more rare than having just a primitive prime factor. At first sight it might seem surprising that one can prove that esoteric dynamical recurrences have super-primitive prime factors whereas second-order linear recurrences (like the Fibonacci numbers) do not. However the numerator and denominator of the $n$th term of a degree- $d$ dynamical recurrence grow like $C^{d^{n}}$, far faster than the $C^{n}$ of linear recurrences, so we might expect each new term to have a much better chance of having a prime factor that we have not seen before. One approach to proving this is simply based on size, the approach used for second-order linear recurrences, and so one might believe it should work even more easily here - this is the approach, for instance, of [5]. Our approach uses simple considerations to imply that a new term in the sequence (or a suitable factor of that term) can only include "old" prime factors from a finite set, and then we use the Thue/Mahler theorem to show that this can happen only finitely often. One further upshot of our method is that it can be made effective; i.e., in principle one could give a bound on the size of the set of exceptions in our main theorems. Essentially this amounts to applying Baker's method to obtain an effective form of the Thue/Mahler theorem; see the discussion in $\S 4$ for a few more details.

The remainder of this article is laid out as follows. In the next section, we give a description of the notation used in the paper. In $\$ 3$ we give a lower bound on the number of distinct zeros a rational function can have outside of certain exceptional scenarios; this tool will be used in $\S 4$ to prove Proposition 1 and the analogous result for periodic points. As an application we deduce a new unified proof that there are infinitely many primes congruent to 1 modulo a fixed odd prime power. In $\S 5, \S 7$ and $\S 8$ we study properties of fixed points and preperiodic points in order to determine when one can change coordinates and apply Proposition 1, and then we use this analysis in $\S 6$ and $\S 9$ to deduce Theorems 1 and 2. Appendix A contains a number of results from complex dynamics that are used throughout the paper, and Appendix B recalls the classification of the exceptional rational maps arising from Baker's theorem, as stated in $§ 7$.

\section{Notation}

Suppose that $K$ is a number field, with ring of integers $R$. If $S$ is a finite set of nonzero prime ideals of $R$, write $R_{S}$ for the ring of $S$-integers-i.e., the set of all elements 
$a / b \in K$ where $a, b \in R$ and the ideal $(b)$ is divisible only by primes in $S$. One may always enlarge a given set of primes $S$ so that $R_{S}$ is a principal ideal domain [2], Proposition 5.3.6. In that case, any $\alpha \in K$ can be written $\alpha=a / b$ with $a, b \in R_{S}$ and $(a, b)=(1)$; i.e., $a$ and $b$ share no common prime ideal factor in $R_{S}$. Moreover, the greatest common divisor of any two elements $a, b \in R_{S}$, denoted $\operatorname{gcd}(a, b)$, is defined to be a generator of the ideal $(a, b)$. It is well defined up to a unit in $R_{S}$.

We will say that a prime ideal $P$ of $K$ divides the numerator (resp. denominator) of an element $\alpha \in K$ to mean that upon writing the fractional ideal $(\alpha)$ as $\mathfrak{a} / \mathfrak{b}$ with $\mathfrak{a}$ and $\mathfrak{b}$ coprime integral ideals, the ideal $P$ divides $\mathfrak{a}$ (resp. $\mathfrak{b}$ ). When $K=\mathbb{Q}$ and $P$ is a rational prime number, this agrees with standard usage.

For any field $k$, we identify the projective space $\mathbb{P}^{1}(k)$ with $k \cup\{\infty\}$. Returning to the number field $K$ with ring of integers $R$, fix a non-zero prime ideal $P \subset R$. Write $R_{P}$ for the localization of $R$ at $P$; the ring $R_{P}$ is the subset of all elements $a / b \in K$ such that $a, b \in R$ and $b \notin P$. The ring $R_{P}$ has a unique maximal ideal $P R_{P}=\{a / b \in K: a \in P, b \notin P\}$, and we can identify the residue fields $R / P R \cong R_{P} / P R_{P}$. There is a canonical reduction map $\mathbb{P}^{1}(K) \rightarrow \mathbb{P}^{1}\left(R_{P} / P R_{P}\right)$ given by sending $\alpha \in R_{P}$ to its image in the quotient $R_{P} / P R_{P}$, and by sending $\alpha \in \mathbb{P}^{1}(K) \backslash R_{P}$ to $\infty$. We extend the notion of congruences modulo $P$ to $\mathbb{P}^{1}(K)$ by saying that $\alpha \equiv \beta(\bmod P)$ if and only if $\alpha$ and $\beta$ have the same canonical reduction in $\mathbb{P}^{1}\left(R_{P} / P R_{P}\right)$. This gives the usual notion of congruence when restricted to $R_{P}$; i.e., $\alpha-\beta \in P R_{P}$ if and only if $\alpha$ and $\beta$ have the same canonical reduction in $\mathbb{P}^{1}\left(R_{P} / P R_{P}\right)$.

For a given $\phi(t) \in K(t)$, fix polynomials $f(t), g(t) \in R[t]$ with no common root in $\bar{K}$ such that $\phi(t)=f(t) / g(t)$. Let $d=\operatorname{deg} \phi:=\max \{\operatorname{deg} f, \operatorname{deg} g\}$; then $F(x, y)=y^{d} f(x / y)$, $G(x, y)=y^{d} g(x / y) \in R[x, y]$ are homogeneous of degree $d$ with no common linear factor over $\bar{K}$. For $F_{0}(x, y)=x, G_{0}(x, y)=y$, we define $F_{r+1}(x, y)=F\left(F_{r}(x, y), G_{r}(x, y)\right)$ and $G_{r+1}(x, y)=G\left(F_{r}(x, y), G_{r}(x, y)\right)$ for all $r \geqq 0$. The polynomials $F_{r}(x, y)$ and $G_{r}(x, y)$ have no common factor in $\bar{K}[x, y]$.

Throughout we will use the notation $\left\{x_{n}\right\}_{n \geqq 0}$ to denote a sequence of elements of $K$ obtained by choosing $x_{0} \in K$ and setting $x_{n+1}=\bar{\phi}\left(x_{n}\right)$ for $n \geqq 0$. We will also write $\phi^{(n)}$ for the $n$-fold composition of $\phi$ with itself, so that $x_{n}=\phi^{(n)}\left(x_{0}\right)$.

\section{Rational functions with many distinct zeros}

Define $\mathscr{T}$ to be the set of rational functions $\phi(t) \in \mathbb{C}(t)$ of degree $d \geqq 2$ of one of the following forms:

(i) $\phi(t)=t^{d} / g(t)$ where $g(t)$ is a polynomial of degree $\leqq d$;

(ii) $\phi(t)=c / t^{d}$ for some constant $c \neq 0$; or

(iii) $\phi(t)=\frac{\alpha(t-\alpha)^{d}}{(t-\alpha)^{d}-c t^{d}}$ for some constants $\alpha, c \neq 0$.

Note that 0 is periodic in a period of length one in (i), and in a period of length two in (ii) and (iii) (where $\phi(0)=\infty$ and $\alpha$, respectively). Geometrically speaking, $\mathscr{T}$ consists of all rational functions of degree $d$ such that $\phi^{(2)}(t)$ has a totally ramified fixed point at 0 . 
The main reason for defining $\mathscr{T}$ is seen in the following lemma:

Lemma 1. Suppose that $\phi(t) \in \mathbb{C}(t) \backslash \mathscr{T}$ has degree $d \geqq 2$. If $r \geqq 4$, then $F_{r}(x, y)$ has at least three non-proportional linear factors.

Proof. [12], Proposition 3.44 states that if $\psi(t) \in \mathbb{C}(t)$ has degree $d \geqq 2$, and if $\psi^{(2)}(t)$ is not a polynomial, then $\psi^{-4}(\infty)$ contains at least three elements. Let $\psi(t)=1 / \phi(1 / t)$ so that $\psi^{(2)}(t) \in \mathbb{C}[t]$ if and only if $\phi^{(2)}(t)$ is of the form $t^{D} / g_{2}(t)$ where $g_{2}(t)$ is a non-zero polynomial of degree $\leqq D=d^{2}$. The result follows by showing that this occurs if and only if $\phi(t) \in \mathscr{T}$ : One easily confirms that, for each $\phi \in \mathscr{T}$, one has $\phi^{(2)}(t)=t^{D} / g_{2}(t)$ for some polynomial $g_{2}(t)$ of degree $\leqq D$. On the other hand, any $\phi^{(2)}(t)$ of this form is totally ramified over 0 . Let $\beta=\phi(0)$ so that $0=\phi(\beta)$. As ramification indices are multiplicative, we deduce that $\phi$ is totally ramified at 0 and at $\beta$. An easy calculation then confirms that the cases $\beta=0, \infty$, or $\alpha(\neq 0$ or $\infty)$ correspond to the three cases in $\mathscr{T}$.

We deduce the following from Lemma 1 :

Corollary 1. Suppose that $\phi(t) \in \mathbb{C}(t) \backslash \mathscr{T}$ has degree $d \geqq 2$. If $r \geqq 4$, then $F_{r}(x, y)$ has at least $d^{r-4}+2$ non-proportional linear factors.

To prove Corollary 1 we use the $a b c$-Theorem for polynomials (see, e.g., [4], Theorem F.3.6). As we will have use for it again later in the paper, we recall the statement:

abc-Theorem for polynomials. If $a, b, c \in \mathbb{C}[x, y]$ are homogeneous forms of degree $d \geqq 1$ with no common linear factor such that $a(x, y)+b(x, y)+c(x, y)=0$, then the number of non-proportional linear factors of abc is at least $d+2$.

Proof of Corollary 1. We have $F_{r}(x, y)=F_{4}(X, Y)$ where $X=F_{r-4}(x, y)$, $Y=G_{r-4}(x, y)$ (which have degree $\left.d^{r-4}\right)$. By Lemma $1, F_{4}(X, Y)$ has at least three nonproportional linear factors in $X, Y$ which must themselves satisfy a linear equation with constant coefficients. Indeed, if the three linear factors are $X-\alpha Y, X-\beta Y$, and $X-\gamma Y$, then

$$
(\beta-\gamma)(X-\alpha Y)+(\gamma-\alpha)(X-\beta Y)+(\alpha-\beta)(X-\gamma Y)=0 .
$$

If the three linear factors are instead $X-\alpha Y, X-\beta Y$, and $Y$, then we use

$$
(X-\alpha Y)-(X-\beta Y)+(\alpha-\beta) Y=0 .
$$

The $a b c$-theorem for polynomials then implies that there are at least $d^{r-4}+2$ coprime linear factors of $(X-\alpha Y)(X-\beta Y)(X-\gamma Y)$, and hence of $F_{4}(X, Y)=F_{r}(x, y)$.

\section{Preperiodic points and super-primitivity}

We use the following result from Diophantine approximation:

The Thue/Mahler Theorem. Suppose that $F(x, y) \in K[x, y]$ is homogeneous and has at least three non-proportional linear factors (over $\bar{K}$ ). Let $S$ be any finite set of primes of $K$. 
There are only finitely many $m / n \in K \backslash\{0\}$ such that all prime factors of $F(m, n)$ belong to the set $S$.

See [2], Theorem 5.3.2 for a proof. ${ }^{2)}$ With this tool in hand we can complete the proof of Proposition 1:

Proof of Proposition 1. Write $y_{0}=0$ and $y_{k+1}=\phi\left(y_{k}\right)$ for each $k \geqq 0$. Let $r_{0}=0$, $s_{0}=1$, and choose $r_{k}, s_{k} \in R$ - the ring of integers of $K$ - so that $y_{k}=r_{k} / s_{k}$ for each $k \geqq 0$. If $y_{k}=\infty$, let $r_{k}=1, s_{k}=0$. As 0 is preperiodic, we may assume there are only finitely many elements $r_{k}$ and $s_{k}$. Let $S$ be the set of prime ideals that either divide $r_{k}$ for some $k \geqq 1$ or that divide $\operatorname{Resultant}(F, G)$. Note that $S$ is finite since $r_{j} \neq 0$ for all $j \geqq 1$, and $f$ and $g$ have no common root in $\bar{K}$. We may also enlarge the set $S$ so that the ring of $S$-integers $R_{S}$ is a principal ideal domain.

Let $P$ be a prime ideal that is not in $S$, and divides the rational prime $p$, so that $R / P R \cong \mathbb{F}_{q}$ for $q$ some power of $p$. Then $\mathbb{P}^{1}(R / P R) \cong \mathbb{P}^{1}\left(\mathbb{F}_{q}\right)=\mathbb{F}_{q} \cup\{\infty\}$. We identify $P$ with the prime ideal $P R_{S}$ in $R_{S}$. There is a canonical isomorphism $R / P R \cong R_{S} / P R_{S}$, and hence also $\mathbb{P}^{1}(R / P R) \cong \mathbb{P}^{1}\left(R_{S} / P R_{S}\right)$.

At most one term of the sequence $\left\{x_{n}\right\}_{n \geq 0}$ is equal to $\infty$, so we may assume that $n$ is large enough that $x_{n} \neq \infty$. Write each $x_{n}=u_{n} / v_{n} \in K$ with $u_{n}, v_{n} \in R_{S}$ and $\left(u_{n}, v_{n}\right)=(1)$. Suppose $P \mid u_{n}$. Observe that since $P$ does not divide $\operatorname{Resultant}(F, G)$, for any $k \geqq 0$ we find that

$$
x_{n+k}=\phi^{(k)}\left(x_{n}\right) \equiv \phi^{(k)}(0)=y_{k} \quad(\bmod P) .
$$

We deduce that if a prime ideal $P$ is not in $S$, then $P$ divides at most one $u_{n}$. For if $P$ divides $u_{m}$ and $u_{n}$ with $n>m$, then $P \mid r_{k}$ with $k=n-m$ by the previous paragraph. Hence $P \in S$, which is a contradiction.

Let $\mathscr{N}$ be the set of integers $n \geqq 0$ such that all prime factors of $u_{n}$ are in the set $S$. Now $F_{4}\left(u_{n-4}, v_{n-4}\right)$ equals $u_{n} \times \operatorname{gcd}\left(F_{4}\left(u_{n-4}, v_{n-4}\right), G_{4}\left(u_{n-4}, v_{n-4}\right)\right)$ so that if $n \in \mathscr{N}$ then all prime factors of $F_{4}\left(u_{n-4}, v_{n-4}\right)$ are in the set $S$. Note that $\phi(t) \notin \mathscr{T}$ as 0 is not a periodic point. Hence, by Lemma $1, F_{4}$ has at least three non-proportional linear factors, and therefore, by the Thue/Mahler Theorem, there are only finitely many $u_{n-4} / v_{n-4} \in K$ such that all prime factors of $F_{4}\left(u_{n-4}, v_{n-4}\right)$ belong to the set $S$. We deduce that there are only finitely many $n \in \mathscr{N}$, since $\left\{x_{n}\right\}_{n \geqq 0}$ is not eventually periodic.

Finally, if $m \notin \mathscr{N}$, then $u_{m}$ has a prime ideal factor $P_{m}$ not in $S$, and we have seen that $P_{m}$ cannot divide $u_{n}$ for any $n \neq m$. Moreover, $P_{m}$ cannot divide $v_{m}$ since $\left(u_{m}, v_{m}\right)=(1)$.

Finally we prove a version of Proposition 1 in the case that 0 is periodic. It is also a special case of [5], Theorem 7, again with the added benefit that our method can be made effective.

2) Although we will not need it at present, the Thue/Mahler Theorem can be made effective. One reduces its proof to the solution of a unit equation, and unit equations are effectively solvable by Baker's method. See [2], $\S 5.4$ for a discussion. 
Proposition 1'. Let $K$ be a number field. Suppose that $\phi(t) \in K(t) \backslash \mathscr{T}$ has degree $d \geqq 2$, and that 0 is a periodic point. If the sequence of $K$-rationals $\left\{x_{n}\right\}_{n \geqq 0}$ is not eventually periodic, then the numerator of $x_{n}$ has a primitive prime factor $P_{n}$ for all sufficiently large $n$.

Remark 1. It is not hard to see that if $\phi \in \mathscr{T}$, then the conclusion of Proposition $1^{\prime}$ does not follow. Indeed a prime dividing $x_{n}$ for some $n \geqq 0$, divides $x_{0}$ in $\mathscr{T}(\mathrm{i})$, and $x_{\delta}$ in $\mathscr{T}$ (ii) and $\mathscr{T}$ (iii) where $\delta$ is the least non-negative residue of $n(\bmod 2)$.

Remark 2. In the proof of Proposition $1^{\prime}$ we actually construct a sequence $\left\{u_{n}^{*}\right\}_{n \geq 0}$ of algebraic integers of $K$, where the numerators $u_{n}$ of $x_{n}$ are a product of powers of $u_{m}^{*}$ with $m \leqq n$; and we show that $u_{n}^{*}$ has a super-primitive prime factor $P_{n}$ for all sufficiently large $n$. Proposition $1^{\prime}$ then follows.

Remark 3. Ingram and Silverman [5] conjecture that if $\phi(t) \in K(t)$ has degree $d \geqq 2$, and if 0 is not a preperiodic point, then for the sequence of $K$-rationals $\left\{x_{n}\right\}_{n \geq 0}$ starting with $x_{0}=0$, the numerator of $x_{n}$ has a primitive prime factor for all sufficiently large $n$. This does not seem approachable using our methods.

Proof of Proposition 1'. This is largely based on the above proof when 0 is preperiodic and not periodic, but has some additional complications. Suppose that 0 has period $q$. Then $F_{q}(x, y)=x^{b} F_{q}^{*}(x, y)$ for some integer $b \geqq 1$ and $F_{q}^{*}(x, y) \in R[x, y]$ where $F_{q}^{*}(0,1) \neq 0$. Therefore if $n>q$ then

$$
F_{n}(x, y)=F_{q}\left(F_{n-q}(x, y), G_{n-q}(x, y)\right)=\left(F_{n-q}(x, y)\right)^{b} F_{q}^{*}\left(F_{n-q}(x, y), G_{n-q}(x, y)\right) .
$$

So we define $F_{n}^{*}(x, y)=F_{n}(x, y) / F_{n-q}(x, y)^{b} \in R[x, y]$, and we let $F_{n}^{*}(x, y)=F_{n}(x, y)$ if $n<q$. Note that

$$
F_{n}(x, y)=\prod_{0 \leqq j \leqq[n / q]} F_{n-j q}^{*}(x, y)^{b^{j}} .
$$

Write $y_{0}=0$ and $y_{k+1}=\phi\left(y_{k}\right)$ for each $k \geqq 0$. Let $S$ be the set of primes of $K$ that either divide the numerator of $y_{k}$ for some $y_{k} \neq 0$, or that divide $\operatorname{Resultant}(F, G)$, or that divide $F_{q}^{*}(0,1)$. This set is finite since 0 is periodic. Enlarge the set $S$ if necessary so that the ring of $S$-integers $R_{S}$ is a PID. Write $y_{k}=r_{k} / s_{k} \in K$ with $r_{k}, s_{k} \in R_{S}$ and $\left(r_{k}, s_{k}\right)=(1)$. There are only finitely many elements $r_{k}$ and $s_{k}$, since 0 is periodic.

Now write each $x_{k}=u_{k} / v_{k} \in K$ with $u_{k}, v_{k} \in R_{S}$ and $\left(u_{k}, v_{k}\right)=(1)$. Suppose that the prime $P$ is not in $S$ and that $m$ is the smallest nonnegative integer such that $P$ divides $F_{m}^{*}\left(u_{0}, v_{0}\right)$. Then $m$ is the smallest integer such that $P$ divides $F_{m}\left(u_{0}, v_{0}\right)$, since $F_{m}(x, y)$ is (as we saw above) the product of $F_{r}^{*}(x, y)$ to various powers, over $r \leqq m$.

Proceeding as in the proof of Proposition 1 , for $P \notin S$ we see that

$$
\phi^{(n)}\left(x_{0}\right)=x_{n}=\phi^{(n-m)}\left(x_{m}\right) \equiv \phi^{(n-m)}(0)=y_{n-m} \quad(\bmod P) .
$$

Hence $P$ divides $F_{n}\left(u_{0}, v_{0}\right)$ if and only if $P$ divides the numerator of $y_{n-m}$, which holds if and only if $q$ divides $n-m$ as $P \notin S$. So if $P$ divides $F_{n}^{*}\left(u_{0}, v_{0}\right)$, we must have that $q$ divides $n-m$, and $n \geqq m$. If $n>m$, let $X=F_{n-q}\left(u_{0}, v_{0}\right), Y=G_{n-q}\left(u_{0}, v_{0}\right)$. As $q$ divides $n-m$ we 
know that $q$ divides $(n-q)-m$, so that $P$ divides $F_{n-q}\left(u_{0}, v_{0}\right)=X$, and hence not $Y$ (else $P$ divides $\operatorname{Resultant}(F, G)$ which implies that $P \in S$, a contradiction). Now

$$
F_{n}^{*}\left(u_{0}, v_{0}\right)=F_{q}^{*}(X, Y) \equiv F_{q}^{*}(0, Y) \equiv Y^{\ell} F_{q}^{*}(0,1) \quad(\bmod P),
$$

where $\ell=\operatorname{deg} F_{q}^{*}$. But $P \nmid Y^{\ell} F_{q}^{*}(0,1)$ as $P \notin S$, and so $P$ does not divide $F_{n}^{*}\left(u_{0}, v_{0}\right)$. Hence we have proved that if $P \notin S$, then there is at most one value of $n$ for which $P$ divides $F_{n}^{*}(x, y)$.

If $F_{q}(X, Y)$ has at least four non-proportional linear factors, then $F_{q}(X, Y) / X^{b}$ has at least three non-proportional linear factors. Now $F_{n}^{*}(x, y)=F_{q}(X, Y) / X^{b}$ where $X=F_{n-q}(x, y), Y=G_{n-q}(x, y)$, and the result follows from the Thue/Mahler Theorem, as in Proposition 1. (Note that the fact that $\phi(t) \notin \mathscr{T}$ is part of the hypothesis.)

If $F_{q}(X, Y)$ has not more than three non-proportional linear factors, select $k \geqq 2$ minimal such that $F_{k q}(x, y)$ has at least $2 k+2$ non-proportional linear factors. We know that such a $k$ exists since Corollary 1 implies that $F_{8 q}(x, y)$ has at least

$$
d^{8 q-4}+2 \geqq 2^{4}+2=2 \cdot 8+2
$$

non-proportional linear factors. Then $F_{k q}(X, Y) / F_{(k-1) q}(X, Y)^{b}$ has at least three nonproportional linear factors. Now we see that $F_{n}^{*}(x, y)=F_{k q}(X, Y) / F_{(k-1) q}(X, Y)^{b}$ where $X=F_{n-k q}(x, y), Y=G_{n-k q}(x, y)$, and the result follows from the Thue/Mahler Theorem, as in Proposition 1.

Remark 4. Propositions 1 and $1^{\prime}$ imply the main results of [11], although no Diophantine approximation was necessary in the cases presented there.

Proposition $1^{\prime}$ gives a unified means for finding prime numbers in certain residue classes.

Application. Let $q^{n}$ be an odd prime power. There exist infinitely many primes of the form $q^{n} k+1$.

Proof. Consider the polynomial $\phi(t)=(t-1)^{q}+1$. Then $\phi(0)=0$ so that 0 is a fixed point, but $\phi \notin \mathscr{T}$. Let $x_{0}$ be any integer larger than 1 . Clearly $x_{m} \rightarrow \infty$ as $m \rightarrow \infty$, and so Proposition 1' implies, after a small shift in notation, that $x_{n+m}$ has an odd primitive prime factor $p_{m}$ for all sufficiently large $m$. By the definition of $\phi$, we see that $x_{m+r}-1=\left(x_{m+r-1}-1\right)^{q}$ for every $r \geqq 1$, and so by induction,

$$
\left(x_{m}-1\right)^{q^{n}}=x_{m+n}-1 \equiv-1 \quad\left(\bmod p_{m}\right) .
$$

That is, $x_{m}-1$ has order dividing $2 q^{n}$ in the group $\left(\mathbb{Z} / p_{m} \mathbb{Z}\right)^{\times}$. If the order of $x_{m}-1$ is $q^{j}$ for some $j \leqq n$, then

$$
-1 \equiv\left(x_{m}-1\right)^{q^{n}}=\left(\left(x_{m}-1\right)^{q^{j}}\right)^{q^{n-j}} \equiv 1 \quad\left(\bmod p_{m}\right),
$$

a contradiction. On the other hand, if the order of $x_{m}-1$ is $2 q^{j}$, then

$$
\left(x_{m}-1\right)^{q^{j}} \equiv-1 \quad\left(\bmod p_{m}\right) \Rightarrow x_{m+j}=\left(x_{m}-1\right)^{q^{j}}+1 \equiv 0 \quad\left(\bmod p_{m}\right),
$$


which contradicts the primitivity of $p_{m}$ unless $j=n$. Hence $x_{m}-1$ has order exactly $2 q^{n}$ in the group $\left(\mathbb{Z} / p_{m} \mathbb{Z}\right)^{\times}$, and consequently $2 q^{n}$ divides $p_{m}-1$. That is, $p_{m} \equiv 1\left(\bmod q^{n}\right)$. Varying $m$, we produce infinitely many primes of this form.

\section{Dynamical systems with exceptional behavior at $\infty$, Part I}

In order to prove Theorem 1, we choose a point that falls into a cycle of length dividing $\Delta$ after exactly one step. The following lemma tells us this is always possible:

Lemma 2. Suppose $\phi(t) \in \mathbb{C}(t)$ has degree $d \geqq 2$ and that $\Delta \geqq 1$ is an integer. There exists a point $\alpha \in \mathbb{P}^{1}(\mathbb{C})=\mathbb{C} \cup\{\infty\}$ such that $\phi^{(\Delta)}(\phi(\alpha))=\phi(\alpha)$, but $\phi^{(\Delta)}(\alpha) \neq \alpha$.

Proof. Suppose not. If $\beta$ is a fixed point of $\phi^{(\Delta)}$, and if $\phi(\gamma)=\beta$ then $\gamma$ is also a fixed point of $\phi^{(\Delta)}$, or else we may take $\alpha=\gamma$. But then $\gamma=\phi^{(\Delta-1)}(\phi(\gamma))=\phi^{(\Delta-1)}(\beta)$ is unique, and so $\beta$ is totally ramified for $\phi$. By symmetry, $\phi(\beta)$ is totally ramified too.

In particular, this implies that $\phi^{\prime}(\beta)=0$ and so $\left(\phi^{(\Delta)}(\beta)\right)^{\prime}=\prod_{j=0}^{\Delta-1} \phi^{\prime}\left(\phi^{(j)}(\beta)\right)=0$. Hence $\beta$ has multiplicity 1 as a root of $\phi^{(\Delta)}(x)-x$ by Lemma A.1. Therefore $d^{\Delta}+1$, the number of fixed points of $\phi^{(\Delta)}(x)-x$, by Lemma A.2, equals the number of such $\beta$, which is not more than the number of totally ramified points of $\phi$. This is at most 2 , by the Riemann/Hurwitz formula (for any map $\phi$ ) and so we have established a contradiction.

Define $\mathscr{E}$ to be the class of rational functions $\phi(t) \in \mathbb{C}(t)$ of degree $d \geqq 2$ satisfying one of the following:

(i) $\phi(t)=t+\frac{1}{g(t)}$ for some polynomial $g(t)$ with $\operatorname{deg}(g)=d-1$;

(ii) $\phi=\sigma^{-1} \circ \psi \circ \sigma$ for some linear transformation $\sigma(t)=\lambda t+\beta$ with $\lambda \neq 0$, where $\psi(t)=\frac{t^{d}}{t^{d-1}+1}$; or

(iii) $\phi=\sigma^{-1} \circ \psi \circ \sigma$ with $\sigma$ as in (ii) and $\psi(t)=\frac{t^{2}}{2 t+1}$.

Observe that in the classes $\mathscr{E}($ ii) and $\mathscr{E}$ (iii), the map $\sigma$ has no denominator, which means that $\infty$ is fixed under the transformation $\sigma$. The point at infinity plays an important role in the appearance of prime divisors in the numerators of our dynamical sequences.

Lemma 3. Let $\phi(t) \in \mathbb{C}(t)$ be a rational function of degree $d \geqq 2$ and $\Delta$ a positive integer. If $\Delta \geqq 2$, or if $\Delta=1$ and $\phi(t) \notin \mathscr{E}$, then there exists $\alpha \in \mathbb{P}^{1}(\mathbb{C})$ such that $\phi^{(\Delta)}(\phi(\alpha))=\phi(\alpha), \phi^{(\Delta)}(\alpha) \neq \alpha$ and $\phi(\alpha) \neq \infty$.

Proof. We proceed as in the proof of Lemma 2: Suppose that the result is false. If $\beta \neq \infty$ is a fixed point of $\phi^{(\Delta)}$, then $\beta$ is totally ramified for $\phi$, and $\phi^{-1}(\beta)$ is another fixed point of $\phi^{(\Delta)}$. Moreover $\beta^{-1}(\beta)$ is a fixed point of multiplicity one for $\phi^{(\Delta)}$ by Lemma A.1. So if there are $r$ finite fixed points of $\phi$, and $R$ finite fixed points of $\phi^{(\Delta)}$, then $r \leqq R \leqq 2$ by 
the Riemann/Hurwitz formula (using the fact that the fixed points of $\phi$ are a subset of the fixed point of $\left.\phi^{(\Delta)}\right)$. If $\infty$ has multiplicity $k$ as a fixed point of $\phi$, then Lemma A.2 gives

$$
k+r=d+1 .
$$

We deduce that $k=d+1-r \geqq 2+1-2=1$; that is, $\infty$ must be a fixed point of $\phi$, and hence of $\phi^{(\Delta)}$. So either the finite fixed points of $\phi^{(\Delta)}$ are also fixed points of $\phi$, or there are two finite fixed points $\beta, \gamma$ of $\phi^{(\Delta)}$ such that $\phi(\beta)=\gamma$ and $\phi(\gamma)=\beta$. In either case we know that the finite fixed points of $\phi^{(\Delta)}$ are totally ramified for $\phi$.

Now suppose that we have two finite fixed points $\beta, \gamma$ of $\phi^{(\Delta)}$ and change coordinates so that $\beta \mapsto 0, \gamma \mapsto \infty$, and $\infty \mapsto 1$ to obtain a new function $\psi$. (When we say "change coordinates so that $a \mapsto b$ ", we mean "replace $\phi$ with $\psi=\sigma^{-1} \circ \phi \circ \sigma$ ", where $\sigma$ is a fractional linear transformation such that $\sigma^{-1}(a)=b$. Then $a$ is a fixed point of $\phi$ if and only if $b$ is a fixed point of $\psi$.) Now $\psi$ is totally ramified at 0 and $\infty$, with pre-images 0 and $\infty$, and $\psi(1)=1$, so that $\psi(t)=t^{ \pm d}$. As $\psi^{\prime}(1) \neq 1$, we see that 1 is a fixed point of $\psi$ of multiplicity 1. Counting fixed points of $\psi$ using Lemma A.2 and the fact that $r$ is the number of fixed points distinct from 1 gives $3 \leqq 1+r=d+1 \leqq 3$; that is, $r=d=2=R$. Now $\psi^{(\Delta)}(t)=t^{2^{\Delta}}$ (as 0 and $\infty$ must be fixed), which has $2^{\Delta}+1$ distinct fixed points, so that $2^{\Delta}+1=1+R=3$, and so $\Delta=1$. Hence the only possibility is $\psi(t)=t^{2}$, and we obtain $\mathscr{E}\left(\right.$ iii) as $\sigma^{-1} \circ \psi \circ \sigma$ with $\sigma(t)=t /(t+1)$. Note that the coordinate change at the beginning of this paragraph moved $\infty \mapsto 1$ and then this last coordinate change sent $1 \mapsto \infty$, so that $\infty$ was not moved in their composition. This corresponds to a change of coordinates of the form $\sigma(t)=\lambda t+\beta$ for some $\lambda \neq 0$.

Henceforth we may assume $R \leqq 1$, so that the multiplicity of $\infty$ as a fixed point of $\phi$ satisfies $k=d+1-r \geqq d+1-R \geqq 2$. By Lemma A.3(1), we know $\infty$ has multiplicity $k$ as a fixed point of $\phi^{(\Delta)}$, and so $d^{\Delta}+1$, the number of fixed points of $\phi^{(\Delta)}$, equals $k+R=d+1-r+R \leqq d+2$. Hence $d\left(d^{\Delta-1}-1\right) \leqq 1$, which implies that $\Delta=1$.

If $R=0$, then $\infty$ is the only fixed point of $\phi$, which means $\phi(t)-t=1 / g(t)$ for some polynomial $g(t)$ of degree $d-1$, from which we obtain $\mathscr{E}(\mathrm{i})$.

If $R=1$, so that $\beta$ is the only finite fixed point of $\phi$, replace $\phi$ with $\phi(t+\beta)-\beta$. Now $\phi(t)=t^{d} / g(t)$, and the numerator of $\phi(t)-t=t\left(t^{d-1}-g(t)\right) / g(t)$ has only one root, so that $g(t)=t^{d-1}+c$ for some constant $c \neq 0$. Taking $\psi(t)=\lambda^{-1} \phi(\lambda t)$ with $\lambda^{d-1}=c$ gives $\mathscr{E}($ ii).

\section{Differences in the terms of dynamical sequences, Part I}

Theorem 1 is closely related to the following result, Theorem $1^{\prime}$, which gives primitive congruences in projective space. Their proofs are almost identical except that we need to be cautious about primes dividing the denominator in order to deduce Theorem 1 . Theorem $1^{\prime}$ is perhaps more aesthetically appealing than Theorem 1 due to the fact that its conclusion holds for every rational function.

Theorem 1'. Suppose that $\phi(t) \in \mathbb{Q}(t)$ has degree $d \geqq 2$, and a positive integer $\Delta$ is given. Let $x_{0} \in \mathbb{Q}$ and define $x_{n+1}=\phi\left(x_{n}\right)$ for each $n \geqq 0$. If the sequence of rationals 
$\left\{x_{n}\right\}_{n \geqq 0}$ is not eventually periodic, then for all sufficiently large $n$ there exists a prime $p_{n}$ such that $x_{n+\Delta} \equiv x_{n}$ in $\mathbb{P}^{1}\left(\mathbb{F}_{p_{n}}\right)$, but $x_{m+\Delta} \neq x_{m}$ in $\mathbb{P}^{1}\left(\mathbb{F}_{p_{n}}\right)$ for any $m<n$.

Proof of Theorem $1^{\prime}$, and then of Theorem 1. Choose a point $\alpha \in \mathbb{P}^{1}(\overline{\mathbb{Q}})$ such that $\phi^{(\Delta)}(\phi(\alpha))=\phi(\alpha)$, but $\phi^{(\Delta)}(\alpha) \neq \alpha$, by Lemma 2. If $\Delta>1$ or if $\phi(t) \notin \mathscr{E}$, then we also insist that $\phi(\alpha) \neq \infty$, by Lemma 3. Note that $\alpha$ is preperiodic, but not periodic for $\phi$.

If $\alpha \in \mathbb{C}$ then define $\psi(t):=\phi(t+\alpha)-\alpha$, else if $\alpha=\infty$ set $\psi(t):=1 / \phi(1 / t)$. Note that 0 is preperiodic, but not periodic for $\psi$ (and so $\psi(t) \notin \mathscr{T}$ ). Note that $\psi(t) \in K(t)$ for some finite Galois extension $K / \mathbb{Q}(\alpha)$.

If $\alpha \in \mathbb{C}$ then let $y_{0}=x_{0}-\alpha$ and $y_{n+1}=\psi\left(y_{n}\right)$ for each $n \geqq 0$; one can easily verify that $y_{n}=x_{n}-\alpha$ for all $n \geqq 0$. If $\alpha=\infty$ then let $y_{0}=1 / x_{0}$ and $y_{n+1}=\psi\left(y_{n}\right)$ for each $n \geqq 0$; now, $y_{n}=1 / x_{n}$ for all $n \geqq 0$.

We apply Proposition 1 to the sequence $\left\{y_{n}\right\}_{n \geq 0}$, so proving that the numerator of $x_{n}-\alpha$ if $\alpha \in \mathbb{C}$, and the denominator of $x_{n}$ if $\alpha=\infty$, has a super-primitive prime factor $P_{n}$ (in $K$ ) for each sufficiently large $n$. By taking $n$ larger if necessary, we may assume that

(i) no $P_{n}$ divides Resultant $(F, G)$ (which guarantees that $\phi(t)$ induces a well-defined map of degree $d$ on $\mathbb{P}^{1}\left(\mathbb{F}_{q}\right)$ by canonically reducing each of its coefficients modulo $P_{n}$, where $\mathbb{F}_{q} \cong R / P_{n} R$ is the finite field with $q$ elements);

(ii) no $P_{n}$ divides the denominator of $\phi(\alpha)$ if $\phi(\alpha) \neq \infty$; and

(iii) $\phi^{(\Delta)}(\alpha) \neq=\left(\bmod P_{n}\right)$ for any $P_{n}$ (where the congruence is taken in $\mathbb{P}^{1}\left(R / P_{n} R\right)$, so that if $\alpha=\infty$ then condition (iii) means that $P_{n}$ does not divide the denominator of $\left.\phi^{(\Delta)}(\infty)\right)$.

We exclude only finitely many prime ideals in this way since $F(x, y)$ and $G(x, y)$ have no common linear factor over $\overline{\mathbb{Q}}$, and since $\alpha$ is not periodic.

The definition of $\alpha$ and the fact that $P_{n}$ divides the numerator of $x_{n}-\alpha$ yields

$$
x_{n+1+\Delta}=\phi^{(\Delta+1)}\left(x_{n}\right) \equiv \phi^{(\Delta+1)}(\alpha)=\phi(\alpha) \equiv \phi\left(x_{n}\right)=x_{n+1} \quad\left(\bmod P_{n}\right)
$$

(i.e., in $\left.\mathbb{P}^{1}\left(R / P_{n} R\right)\right)$. If $p_{n}$ is the rational prime divisible by $P_{n}$ then $x_{n+1+\Delta} \equiv x_{n+1}\left(\bmod p_{n}\right)$, since $x_{n+1+\Delta}-x_{n+1}$ is rational. Note that if $\Delta>1$ or if $\phi(t) \notin \mathscr{E}$, then $\phi(\alpha) \neq \infty$, and so, by condition (ii), $p_{n}$ does not divide the denominator of $x_{n+1}$ or $x_{n+1+\Delta}$.

We claim that $p_{n}$ is a primitive prime factor of $x_{n+1+\Delta}-x_{n+1}$. Indeed, suppose that $p_{n}$ is a factor of $x_{m+\Delta}-x_{m}$ for some $m<n+1$. Then

$$
\begin{aligned}
\phi^{(\Delta)}(\alpha) \equiv \phi^{(\Delta)}\left(x_{n}\right) & =\phi^{(\Delta)}\left(\phi^{(n-m)}\left(x_{m}\right)\right) \\
& =\phi^{(n-m)}\left(x_{m+\Delta}\right) \equiv \phi^{(n-m)}\left(x_{m}\right)=x_{n} \equiv \alpha\left(\bmod P_{n}\right),
\end{aligned}
$$


contradicting the assumption in (iii). We conclude that $p_{n}$ is a primitive prime factor of $x_{n+1+\Delta}-x_{n+1}$ and, changing variable to $N=n+1$, we deduce that there exists a primitive prime factor $p_{n}$ of $x_{N+\Delta}-x_{N}$ for all sufficiently large $N$.

This completes the proof of Theorem 1'. It also finishes the proof of Theorem 1 when $\Delta>1$ or when $\Delta=1$ and $\phi(t) \notin \mathscr{E}$, so it remains to treat the case $\Delta=1$ and $\phi(t) \in \mathscr{E}$. Let $x_{n}=u_{n} / v_{n}$ for coprime integers $u_{n}, v_{n}$. By Theorem $1^{\prime}$, either the numerator of $x_{n+1}-x_{n}$ or $v_{n}$, the denominator of $x_{n}$, has a primitive prime factor $p_{n}$.

Now if $\phi(t)=t+1 / g(t)$ for some polynomial $g(t)$ of degree $d-1$, then

$$
x_{n+1}-x_{n}=\phi\left(x_{n}\right)-x_{n}=\frac{1}{g\left(x_{n}\right)}=\frac{v_{n}^{d-1}}{v_{n}^{d-1} g\left(u_{n} / v_{n}\right)} .
$$

Evidently the numerator is divisible by $p_{n}$, being a power of $v_{n}$, except perhaps if $p_{n}$ divides the leading coefficient of $g$ (which can only occur for finitely many $n$ ). This completes the proof of Theorem 1 for such functions $\phi(t)$.

$$
\begin{aligned}
& \text { Similarly if } \lambda \phi(t)+\beta=\frac{(\lambda t+\beta)^{d}}{(\lambda t+\beta)^{d-1}+1}, \text { so that } \phi \in \mathscr{E}(\mathrm{ii}), \text { then } \\
& \lambda\left(x_{n+1}-x_{n}\right)=\lambda \phi\left(x_{n}\right)-\lambda x_{n}=-\frac{\left(\lambda x_{n}+\beta\right)}{\left(\lambda x_{n}+\beta\right)^{d-1}+1}=-\frac{\left(\lambda u_{n}+\beta v_{n}\right) v_{n}^{d-2}}{\left(\lambda u_{n}+\beta v_{n}\right)^{d-1}+v_{n}^{d-1}},
\end{aligned}
$$

which is divisible by $p_{n}$ when $d>2$ since $v_{n}$ is in the numerator, except perhaps if $p_{n}$ divides the numerator of $\lambda$. (Note that $\beta$ and $\lambda$ need not be rational, but the conclusion follows anyway upon consideration of prime ideal divisors.)

For $d=2$ and $\phi \in \mathscr{E}(\mathrm{ii})$, we study the function $\psi(t)=\frac{t^{2}}{t+1}$, proving that $\psi^{(r+1)}(t)-\psi^{(r)}(t)=-\frac{t^{2^{r}}}{g_{r}(t)}$ where $g_{r}(t)$ is a monic polynomial in $\mathbb{Z}[t]$ of degree $2^{r}$ by induction. For $r=0$ we have this with $g_{0}(t)=t+1$ by definition. For $r \geqq 1$ we have, using the induction hypothesis,

$$
\begin{aligned}
\psi^{(r+1)}(t)-\psi^{(r)}(t) & =\psi^{(r)}(\psi(t))-\psi^{(r-1)}(\psi(t))=-\frac{\psi(t)^{2^{r-1}}}{g_{r-1}(\psi(t))} \\
& =-\frac{t^{2^{r}}}{(t+1)^{2^{r-1}} g_{r-1}\left(\frac{t^{2}}{t+1}\right)}=-\frac{t^{2^{r}}}{g_{r}(t)} .
\end{aligned}
$$

Hence the prime divisors of the numerator of $x_{r+1}-x_{r}$ are always the same: namely the prime divisors of the numerator of $x_{0}$. Similarly, for $\phi(t)$ obtained through the linear transformation $t \mapsto \lambda t+\beta$, the prime factors of the numerator of $x_{r+1}-x_{r}$ are always the same, namely the prime divisors of the numerator of $\lambda x_{0}+\beta$ and the prime divisors of the denominator of $\lambda$.

Finally, we show that the rational functions $\mathscr{E}\left(\right.$ iii) are exceptional: For $\psi(t)=\frac{t^{2}}{2 t+1}$ we observe that $1+\frac{1}{\psi(t)}=\left(1+\frac{1}{t}\right)^{2}$, and so $1+\frac{1}{\psi^{(r)}(t)}=\left(1+\frac{1}{t}\right)^{2^{r}}$ by an appropriate 
induction hypothesis. Hence

$\psi^{(r)}(t)=\frac{1}{\left(1+\frac{1}{t}\right)^{2^{r}}-1}$ and $\psi^{(r+1)}(t)-\psi^{(r)}(t)=-\frac{\left(1+\frac{1}{t}\right)^{2^{r}}}{\left(1+\frac{1}{t}\right)^{2^{r+1}}-1}=\frac{(t(t+1))^{2^{r}}}{t^{2^{r+1}}-(t+1)^{2^{r+1}}}$.

Therefore the prime divisors of the numerator of $x_{r+1}-x_{r}$ are always the same, namely the prime divisors of the numerator of $x_{0}\left(x_{0}+1\right)$. Similarly, for $\phi(t)$ obtained through the linear transformation $t \mapsto \lambda t+\beta$, the prime factors of the numerator of $x_{r+1}-x_{r}$ are always the same, namely the prime divisors of the numerator of $\left(\lambda x_{0}+\beta\right)\left(\lambda x_{0}+\beta+1\right)$ and those of the denominator of $\lambda$.

Remark 5. It is desirable to remove the Thue/Mahler Theorem from the above proof, because, even though it is effective, the constants that come out are so large as to be of little practical use. Moreover, the constants should grow with the field of definition of $\alpha$ (as chosen in the proof), and thus one should not expect any strong uniformity in $\Delta$ to come from this argument. So, do we really need the full power of the Thue/Mahler Theorem in this proof? In fact it may be the case that our proof can be modified to show that the exceptional $u_{n}$ must divide a particular non-zero integer (rather than $u_{n}$ only having prime factors from a particular finite set). If we examine the proof above then we see that this idea works fine for the primes $P_{n}$ of types (ii) and (iii). It is the primes that divide that resultant (i.e., those of type (i)) that require careful consideration to determine whether their effect can be understood in this way.

Remark 6. In the introduction we gave the example $x_{0}=1$ with $x_{n+1}=x_{n}^{2} /\left(2 x_{n}+1\right)$ so that $x_{n}=\frac{1}{F_{n}-2}$, and $x_{n+1}-x_{n}=-\frac{2^{2^{n}}}{F_{n+1}-2}$. Another amusing example is given by

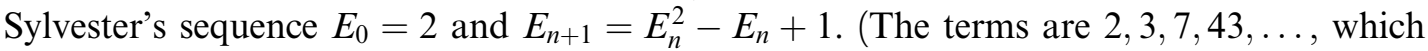
can occur in a version of Euclid's proof of the infinitude of primes, based on the fact that $E_{n}=E_{n-1} E_{n-2} \cdots E_{0}+1$.) Now let $x_{0}=1$ with $x_{n+1}=x_{n}^{2} /\left(x_{n}+1\right)$ so that $x_{n}=\frac{1}{E_{n}-1}$, and $x_{n+1}-x_{n}=-\frac{1}{E_{n}}$, so that there are never prime divisors of the numerator of $x_{n+1}-x_{n}$.

Remark 7. One could instead use Proposition $1^{\prime}$ to prove Theorem 1. In this case, we would have to select a point $\alpha \in \mathbb{P}^{1}(\overline{\mathbb{Q}}) \backslash\{\infty\}$ that is periodic with period dividing $\Delta$, but that is not totally ramified. This allows us to change coordinates to obtain a new rational function that is not in $\mathscr{T}$. One can choose a fixed point $\alpha$ with this property precisely when $\phi \notin \mathscr{E}$, and then the proof proceeds essentially as above.

Remark 8. If $p$ is a prime dividing the numerator of $x_{n+\Delta}-x_{n}$ but not Resultant $(F, G)$, then

$$
x_{n+1+\Delta}=\phi\left(x_{n+\Delta}\right) \equiv \phi\left(x_{n}\right)=x_{n+1} \quad(\bmod p)
$$

and so $p$ divides the numerator of $x_{n+1+\Delta}-x_{n+1}$. Iterating we find that $p$ divides the numerator of $x_{m+\Delta}-x_{m}$ for all $m>n$. 
We can also ask to understand the power of $p$ appearing as a factor in each subsequent term. From the Taylor expansion $\phi(t+h)=\phi(t)+h \phi^{\prime}(t)+\frac{h^{2}}{2 !} \phi^{\prime \prime}(t)+\cdots$ with $h=\phi^{(\Delta)}(t)-t$, we deduce that $\phi^{(\Delta+1)}(t)-\phi(t)-\left(\phi^{(\Delta)}(t)-t\right) \phi^{\prime}(t)$ is divisible by $\left(\phi^{(\Delta)}(t)-t\right)^{2}$. Taking $t=x_{n}$ we deduce that $x_{n+\Delta}-x_{n}$ divides $x_{n+1+\Delta}-x_{n+1}$, up to a bounded quantity, so we recover the result of the previous paragraph. But we can go much further assuming that the numerators of $\phi^{(\Delta)}(t)-t$ and $\phi^{\prime}(t)$ have no common factor. If so, then the gcd of the numerators of $\phi^{(\Delta)}\left(x_{n}\right)-x_{n}$ and $\phi^{\prime}\left(x_{n}\right)$ divides the resultant of the polynomials in the two numerators, which is non-zero. Hence for all but finitely many primes $p_{n}$, if $p_{n}^{e} \| x_{n+\Delta}-x_{n}$ where $p_{n} \nmid x_{m}$ for all $m<n$, then $p_{n}^{e} \| x_{N+\Delta}-x_{N}$ for all $N \geqq n$.

\section{Baker's Theorem and primitive prime factors}

Baker's Theorem ([1], Theorem 3). A rational map of degree $d \geqq 2$ defined over $\mathbb{C}$ has a periodic point in $\mathbb{P}^{1}(\mathbb{C})$ of exact period $\Delta \geqq 2$ except perhaps when $\Delta=2, d=2,3$ or 4 , or when $\Delta=3, d=2$. There exist exceptional maps in each of these four cases.

The exceptional maps in Baker's Theorem are also exceptions to our Theorem 2, as is shown by Lemma 4 below. The exceptions were classified up to conjugation by a linear fractional transformation by Kisaka [7]; see Appendix B for the classification. As in the introduction, let us write $\mathscr{B}_{\Delta, d}$ for the set of rational functions of degree $d$ with no point of exact period $\Delta$; so $\mathscr{B}_{2,2} \cup \mathscr{B}_{2,3} \cup \mathscr{B}_{2,4} \cup \mathscr{B}_{3,2}$ is the set of exceptions in Baker's Theorem.

Lemma 4. Suppose $\phi(t) \in \mathscr{B}_{\Delta, d} \cap \mathbb{Q}(t)$ is a rational map of degree $d$ with no periodic point of period $\Delta$. There exists a finite set of primes $S$ such that for any $u / v \in \mathbb{Q}$, either $\phi(u / v)=\infty, \phi^{(\Delta)}(u / v)=\infty$, or else every $p \notin S$ that divides the numerator of $\phi^{(\Delta)}(u / v)-u / v$ is also a factor of the numerator of $\phi(u / v)-u / v$.

Proof. Write

$$
\begin{array}{r}
\phi(x / y)-x / y=\frac{A_{1}(x, y)}{B_{1}(x, y)}, \\
\phi^{(\Delta)}(x / y)-x / y=\frac{A_{\Delta}(x, y)}{B_{\Delta}(x, y)},
\end{array}
$$

where $A_{i}(x, y), B_{i}(x, y) \in \mathbb{Z}[x, y]$ are homogeneous polynomials such that $A_{1}$ and $B_{1}$ (resp. $A_{\Delta}$ and $B_{\Delta}$ ) share no common linear factor over $\overline{\mathbb{Q}}$ and no common factor in their content. (Recall that the content of a polynomial with integer coefficients is the greatest common divisor of its coefficients.) As $\phi(t)$ has no point of exact period $\Delta$, every solution in $\mathbb{P}^{1}(\overline{\mathbb{Q}})$ to $\phi^{(\Delta)}(\alpha)=\alpha$ must also be a solution to $\phi(\alpha)=\alpha$. (Here we are using the fact that $\Delta=2$ or 3 is prime.) In particular, any non-constant factor of $A_{\Delta}(x, y)$ that is irreducible over $\mathbb{Z}$ is also a factor of $A_{1}(x, y)$.

Define $S$ to be the set of primes dividing Resultant $\left(A_{1}, B_{1}\right)$ together with those primes dividing the content of $A_{\Delta}$. We may assume that $\phi(u / v)$ and $\phi^{(\Delta)}(u / v)$ are not equal to infinity, and also that $u, v$ are coprime integers. Suppose $p \notin S$ is a prime factor of the numerator of $\phi^{(\Delta)}(u / v)-u / v$. Then $p \mid A_{\Delta}(u, v)$, and consequently there exists an 
irreducible factor (over $\mathbb{Z}$ ) of $A_{\Delta}$, say $Q(x, y)$, such that $p \mid Q(u, v)$. By the last paragraph, we know $Q(x, y)$ divides $A_{1}(x, y)$, and hence $p \mid A_{1}(u, v)$. Now $p \nmid B_{1}(u, v)$ since otherwise $p \mid \operatorname{Resultant}\left(A_{1}, B_{1}\right)$. We conclude that $p$ divides the numerator of $\phi(u / v)-u / v$.

Corollary 2. Suppose $(\Delta, d)$ is one of the exceptional pairs in Baker's Theorem, and let $\phi(t) \in \mathscr{B}_{\Delta, d} \cap \mathbb{Q}(t)$. There is a finite set of primes $S$ with the following property. If we define $x_{0} \in \mathbb{Q}$ and $x_{n+1}=\phi\left(x_{n}\right)$, and if the sequence $\left\{x_{n}\right\}_{n \geq 0}$ is not eventually periodic, then for all $n$ sufficiently large, any prime $p \notin S$ that divides the numerator of $x_{n+\Delta}-x_{n}$ must also divide the numerator of $x_{n+1}-x_{n}$.

\section{Dynamical systems with exceptional behavior at infinity, Part II}

Recall that $p_{\Delta, n}$ is a doubly primitive prime factor of $x_{n+\Delta}-x_{n}$ if $p_{\Delta, n}$ divides the numerator of $x_{n+\Delta}-x_{n}$, and if $N \geqq n$ and $D \geqq \Delta$ whenever $p_{\Delta, n}$ divides the numerator of $x_{N+D}-x_{N}$. To produce a doubly primitive prime factor of the numerator of $x_{n+\Delta}-x_{n}$, we want an $\alpha \in \mathbb{P}^{1}(\overline{\mathbb{Q}})$ such that $\phi(\alpha)$ is not $\infty$, and $\phi(\alpha)$ has exact period $\Delta$, while $\alpha$ is not itself periodic. This will allow us to apply Proposition 1 inductively as in the proof of Theorems 1 and $1^{\prime}$.

Lemma 5. Suppose $\phi(t) \in \mathbb{C}(t)$ is a rational function of degree $d \geqq 2$, and let $\Delta \geqq 1$ be an integer. There exists a point $\alpha \in \mathbb{P}^{1}(\mathbb{C})=\mathbb{C} \cup\{\infty\}$ such that $\phi(\alpha)$ has exact period $\Delta$, $\phi(\alpha) \neq \infty$, and $\phi^{(\Delta)}(\alpha) \neq \alpha$ unless

(1) $\Delta=1$ and $\phi(t) \in \mathscr{E}$ (see $§ 5$ for the definition of $\mathscr{E})$; or

(2) $\Delta=2$ and $\phi(t)=\alpha+1 / g(t-\alpha)$ for some $\alpha \in \mathbb{C}$ and some quadratic polynomial $g(t) \in \mathbb{C}[t]$ such that $g(0)=0$, but $g(t) \neq c t^{2}$ for any complex number $c ;$ or

(3) $\Delta=2$ and $\phi=\sigma^{-1} \circ \psi \circ \sigma$ for some $\sigma(t)=(\alpha t+\beta) /(\gamma t+\delta)$ with $\alpha \delta-\beta \gamma \neq 0$, and $\psi(t)=1 / t^{2} ;$ or

(4) $\Delta=2$ and $\phi \in \mathscr{B}_{2, d}$ for some $d=2,3,4$ (see $\S 7$ for the definition of $\left.\mathscr{B}_{\Delta, d}\right)$; or

(5) $\Delta=3$ and $\phi \in \mathscr{B}_{3,2}$.

Proof. Assume that no such $\alpha$ exists. Baker's Theorem states that if $\phi$ has no point of exact period $\Delta$ then we are in case (4) or (5). So henceforth assume that $\phi$ has a point $\gamma$ of exact period $\Delta$. Every point in the orbit of $\gamma$ must also have exact period $\Delta$, and hence there are at least $\Delta$ points of exact period $\Delta$.

As in the proofs of Lemmas 2 and 3 , if $\beta \neq \infty$ has period $\Delta$, then $\beta$ must be totally ramified for $\phi$ else there would be an $\alpha$ as desired with $\phi(\alpha)=\beta$. There are not more than two elements that are totally ramified for (any rational map) $\phi$, hence there can be not more than three points of exact period $\Delta$ (that is, $\infty$ and the two fully ramified points). Hence $\Delta \leqq 3$.

The case $\Delta=1$ is given by Lemma 3 in $\S 5$ : The exceptions are precisely those in $\mathscr{E}$; that is, case (1). 
If $\Delta=2$ or 3 then all of the points of exact order $\Delta$ must be in a unique orbit, else there would be at least $2 \Delta \geqq 4$ points of exact order $\Delta$, a contradiction.

So, if $\Delta=3$ then there is exactly one orbit, containing $\infty$ and two totally ramified points which, we will show, is impossible. We may conjugate by a linear fractional transformation in order to assume the totally ramified points are 0 and 1 , and that the other fixed point is 2 . We suppose that $\phi(2)=1, \phi(1)=0, \phi(0)=2$. The only possible rational function with these ramification conditions is

$$
\phi(t)=\frac{2^{d+1}(t-1)^{d}}{2^{d+1}(t-1)^{d}-(t-2)^{d}},
$$

so that

$$
\phi^{(3)}(t)-t=\phi\left(\phi^{(2)}(t)\right)-t=\frac{(1-t) 2^{d+1}\left(\phi^{(2)}(t)-1\right)^{d}+t\left(\phi^{(2)}(t)-2\right)^{d}}{2^{d+1}\left(\phi^{(2)}(t)-1\right)^{d}-\left(\phi^{(2)}(t)-2\right)^{d}} .
$$

We choose homogeneous polynomials $F_{2}(x, y), G_{2}(x, y) \in \mathbb{C}[x, y]$ of degree $d^{2}=\operatorname{deg} \phi^{(2)}$ with no common linear factor so that $\phi^{(2)}(x / y)=F_{2}(x, y) / G_{2}(x, y)$ after clearing denominators. (One can use the polynomials defined in $\$ 2$, for example.) The numerator of $\phi^{(3)}(x / y)-x / y$ is therefore

$$
N_{3}(x, y)=(y-x) 2^{d+1}\left(F_{2}(x, y)-G_{2}(x, y)\right)^{d}+x\left(F_{2}(x, y)-2 G_{2}(x, y)\right)^{d} .
$$

Dividing through by the common factors $x(x-y)$ we will apply the $a b c$-theorem for polynomials $(\S 3)$. Note that the number of non-proportional linear factors of $N_{3}(x, y)$ is 3 plus the number of distinct fixed points of $\phi(t)$, which is at most $3+(d+1)=d+4$. Hence the total number of distinct roots in our $a b c$-equation is at most $2 \operatorname{deg} \phi^{(2)}+d+4$. The $a b c$ theorem implies that $\left(d \operatorname{deg} \phi^{(2)}-1\right)+2 \leqq 2 \operatorname{deg} \phi^{(2)}+d+4$, hence $d^{2}(d-2) \leqq d+3$, and so $d=2$. When $d=2$ we find that the numerator of $\phi^{(3)}(t)-t$ is

$$
t(t-1)(t-2)\left(7 t^{3}-14 t^{2}+8\right) N_{1}(t)
$$

where $N_{1}(t)$ is the numerator of $\phi(t)-t$. It follows that we have a second cycle of exact order three, consisting of the roots of $7 t^{3}-14 t^{2}+8$. But this contradicts our hypothesis that only one orbit of length 3 exists.

We know that if $\Delta=2$ then there is a single orbit of length 2 . If both points of order 2 are totally ramified, we change coordinates so that the 2 -cycle consists of 0 and $\infty$, and that 1 is a fixed point. Then $\psi(t)=1 / t^{d}$, and hence

$$
\psi(t)-t=\left(1-t^{d+1}\right) / t^{d}, \quad \psi^{(2)}(t)-t=t\left(t^{d^{2}-1}-1\right) .
$$

Since there are no points of exact order 2 other than 0 and $\infty$, all of the $\left(d^{2}-1\right)^{\text {th }}$ roots of unity (which satisfy $\left.\psi^{(2)}(t)=t\right)$ must also be $(d+1)^{\text {th }}$ roots of unity, so as to satisfy $\psi(t)=t$. Hence $d^{2}-1 \leqq d+1$, and thus $d=2$ and $\psi(t)=1 / t^{2}$. It follows that any rational map of the form $\phi(t)=\left(\sigma^{-1} \circ \psi \circ \sigma\right)(t)$ with $\sigma(t)=(\alpha t+\beta) /(\gamma t+\delta)$ and $\alpha \delta-\beta \gamma \neq 0$ has a unique periodic orbit of length 2 consisting entirely of totally ramified points, yielding case (3). 
Finally suppose that $\Delta=2$ and there is a unique cycle of length 2 consisting of $\infty$ and one totally ramified point, $\alpha \in \mathbb{C}$. We may assume $\infty$ is not totally ramified, else we are in the previous case. We have $\phi(t)=\alpha+1 / g(t-\alpha)$ where $\operatorname{deg}(g)=d$ and $g(0)=0$, and $g(t) \neq c t^{d}$ for some complex number $c$. The remainder of the proof follows the strategy of Baker's Theorem. The map $\phi^{(2)}$ has $d^{2}+1$ fixed points counted with multiplicity (Lemma A.2). Two of them are $\alpha$ and $\infty$, which each have multiplicity 1 since the fixed point multiplier of $\phi^{(2)}$ at $\alpha$ and $\infty$ is zero (because $\left(\phi^{(2)}\right)^{\prime}(\alpha)=\left(\phi^{(2)}\right)^{\prime}(\infty)=\phi^{\prime}(\infty) \phi^{\prime}(\alpha)=0$ as $\alpha$ is ramified). All of the remaining fixed points of $\phi^{(2)}$ must be fixed points of $\phi$ as we are assuming there are no other periodic orbits of length 2. By Lemma A.3, each of the remaining fixed points $\beta_{i} \in \mathbb{C}$ falls into exactly one of the following categories:

(i) The fixed point multiplicity of $\beta_{i}$ for $\phi$ and $\phi^{(2)}$ is 1 . Suppose there are $M$ fixed points of this type.

(ii) The fixed point multiplicity of $\beta_{i}$ for $\phi$ is $\ell_{i}>1$, which implies the fixed point multiplicity for $\phi^{(2)}$ is also $\ell_{i}$.

(iii) The fixed point multiplicity of $\beta_{i}$ for $\phi$ is 1 , and the fixed point multiplicity of $\beta_{i}$ for $\phi^{(2)}$ is $2 k_{i}+1$ for some positive integer $k_{i}$. Suppose there are $r$ fixed points of this type.

As $\phi$ has exactly $d+1$ fixed points (always with multiplicity), we may add up the fixed points of these types to find

$$
d+1=M+\sum \ell_{i}+r
$$

Applying the same reasoning to $\phi^{(2)}$ and noting that we must also count 0 and $\infty$, we have

$$
d^{2}+1=2+M+\sum \ell_{i}+\sum\left(2 k_{i}+1\right)=2+M+\sum \ell_{i}+r+2 \sum k_{i}
$$

Subtracting the first of these equations from the second gives $d^{2}-d=2+2 \sum k_{i}$, or $\sum k_{i}=\frac{1}{2}\left(d^{2}-d-2\right)$. On the other hand, Lemma A.3 also tells us that each of the type (iii) fixed points $\beta_{i}$ attracts $k_{i}$ distinct critical points. Since a critical point can only be attracted to a single one of the $\beta_{i}$, we see that there are $\sum k_{i}$ distinct critical points attracted to the set of fixed points $\left\{\beta_{i}\right\}$. By the Riemann/Hurwitz formula, there are exactly $2 d-2$ critical points (with multiplicity). Now $\infty$ is a critical point of order $d-1$ (as $\alpha$ is totally ramified), but it is also a periodic point, so it cannot be attracted to one of the $\beta_{i}$. Hence

$$
\frac{1}{2}\left(d^{2}-d-2\right)=\sum k_{i} \leqq d-1 \Rightarrow d \leqq 3 .
$$

If $d=2$, then $\phi$ has three fixed points counted with multiplicity, and $\phi^{(2)}$ has 5 (Lemma A.2). Hence there are exactly two points of exact period 2, namely $\alpha$ and $\infty$. This yields case (2).

If $d=3$, let us change coordinates so that $\alpha=0$. Now $\phi(t)=1 / g(t)$, where $g(t)=a t^{3}+b t^{2}+c t$ and $a \neq 0$. Choosing $\delta \in \mathbb{C}$ such that $\delta^{4}=a^{-1}$ and replacing $\phi(t)$ by 
$\delta^{-1} \phi(\delta t)$, we may even suppose that $a=1$. A direct calculation shows the numerator of $\phi(t)-t$ is

$$
t^{4}+b t^{3}+c t^{2}-1
$$

while the numerator of $\phi^{(2)}(t)-t$ is

$$
t\left(t^{4}+b t^{3}+c t^{2}-1\right)\left(t^{4}+2 b t^{3}-\left(-b^{2}-c\right) t^{2}+b c t+1\right) .
$$

Therefore $\phi$ has a second periodic orbit of length 2 if the polynomials $t^{4}+b t^{3}+c t^{2}-1$ and $t^{4}+2 b t^{3}-\left(-b^{2}-c\right) t^{2}+b c t+1$ have no common root. The resultant of these two polynomials is $b^{4}-4 b^{2} c+16$, which shows that they have a common root if and only if $b \neq 0$ and $c=\left(b^{4}+16\right) / 4 b^{2}$. Let us now assume that $c=\left(b^{4}+16\right) / 4 b^{2}$, in which case the numerators of $\phi(t)-t$ and $\phi^{(2)}(t)-t$ become

$$
\begin{gathered}
(2 t+b)\left(2 b^{2} t^{3}+b^{3} t^{2}+8 t-4 b\right) \text { and } \\
t(2 t+b)^{3}\left(2 b^{2} t^{3}+b^{3} t^{2}+8 t-4 b\right)\left(4 b^{4} t^{2}+4 t b^{5}+16 b^{2}\right) .
\end{gathered}
$$

The roots of the final factor $4 b^{4} t^{2}+4 t b^{5}+16 b^{2}$ yield a new periodic orbit of length 2 , a contradiction.

Lemma 5 is used in the proof of Theorem 2 as in the following:

Lemma 6. Suppose that $\phi(t) \in \mathbb{Q}(t)$ is a rational function of degree $d \geqq 2$, and that there exists a point $\alpha \in \mathbb{P}^{1}(\overline{\mathbb{Q}})$ such that $\phi(\alpha)$ has exact period $\Delta \geqq 1, \phi(\alpha) \neq \infty$, and $\phi^{(\Delta)}(\alpha) \neq \alpha$. Let $x_{0} \in \mathbb{Q}$ and define $x_{n+1}=\phi\left(x_{n}\right)$ for each $n \geqq 0$, and suppose that the sequence $\left\{x_{n}\right\}_{n \geq 0}$ is not eventually periodic. Suppose that $P$ is a prime ideal that does not divide $\operatorname{Resultant}(f, g)$ (where $\phi=f / g$ ), and that $p$ is the rational prime divisible by $P$. If $P$ divides the numerator of $x_{n}-\alpha$, but neither the denominator of $\alpha$ nor $\phi(\alpha)$, and neither the numerator of $\phi^{(\Delta)}(\alpha)-\alpha$ nor $\phi^{(l)}(\phi(\alpha))-\phi(\alpha)$ for any $1 \leqq l<\Delta$, then the prime $p$ divides the numerator of $x_{N+D}-x_{N}$ if and only if $N \geqq n+1$ and $\Delta$ divides $D$.

Proof. We begin by noting that

$$
x_{n+\Delta+1}=\phi^{(\Delta)}\left(\phi\left(x_{n}\right)\right) \equiv \phi^{(\Delta)}(\phi(\alpha))=\phi(\alpha) \equiv \phi\left(x_{n}\right)=x_{n+1} \quad(\bmod P),
$$

and so $p$ divides the numerator of $x_{n+1+\Delta}-x_{n+1}$. (We have used the fact that $P$ does not divide the denominator of $\phi(\alpha)$.)

If $N \equiv n+j(\bmod \Delta)$ for $1 \leqq j \leqq \Delta$ with $N>n$ then $x_{N} \equiv x_{n+j}(\bmod p)$. To see this, we proceed by induction on $N \geqq n+1+\Delta$ since

$$
x_{N}=\phi^{(N-(n+1+\Delta))}\left(x_{n+1+\Delta}\right) \equiv \phi^{(N-(n+1+\Delta))}\left(x_{n+1}\right)=x_{N-\Delta} \quad(\bmod p) .
$$

We now prove that if $p$ divides the numerator of $x_{N+D}-x_{N}$, then $\Delta$ divides $D$. If not let $D \equiv l(\bmod \Delta)$ where $1 \leqq l<\Delta$, and select $m$ a large integer such that $m \equiv n+1(\bmod \Delta)$. Then, using the congruences of the previous paragraph,

$$
x_{n+1} \equiv x_{m}=\phi^{(m-N)}\left(x_{N}\right) \equiv \phi^{(m-N)}\left(x_{N+D}\right)=x_{m+D} \equiv x_{n+1+l} \quad(\bmod p) .
$$


Hence

$$
\phi(\alpha) \equiv \phi\left(x_{n}\right)=x_{n+1} \equiv x_{n+1+l}=\phi^{(l+1)}\left(x_{n}\right)=\phi^{(l)}(\phi(\alpha)) \quad(\bmod P),
$$

which contradicts the hypothesis. $D$. Then

Finally suppose that $p$ divides the numerator of $x_{N+D}-x_{N}$ with $N \leqq n$ and $\Delta$ divides

$$
x_{n}=\phi^{(n-N)}\left(x_{N}\right) \equiv \phi^{(n-N)}\left(x_{N+D}\right)=x_{n+D} \equiv x_{n+\Delta} \quad(\bmod p)
$$

using the congruence from two paragraphs above, and so

$$
\alpha \equiv x_{n} \equiv x_{n+\Delta}=\phi^{(\Delta)}\left(x_{n}\right) \equiv \phi^{(\Delta)}(\alpha) \quad(\bmod P)
$$

which contradicts the hypothesis.

\section{Differences in the terms of dynamical sequences, Part II}

Now we give the proof of Theorem 2. We recall the statement for the reader's convenience. Define $\mathscr{F}_{1}$ to be those $\phi(t) \in \mathbb{Q}(t)$ of the form $\sigma^{-1} \circ \psi \circ \sigma$, for some linear transformation $\sigma(t)=\lambda t+\beta$ with $\lambda \neq 0$, where

$$
\psi(t)=\frac{t^{2}}{t+1} \text { or } \frac{t^{2}}{2 t+1} .
$$

Define $\mathscr{F}_{2}$ to be the union of $\mathscr{B}_{2, d}$ for $d=2,3,4$ along with all rational maps $\phi(t)$ of the form $\phi=\sigma^{-1} \circ \psi \circ \sigma$ for some $\sigma(t)=(\alpha t-\beta) /(\gamma t-\delta)$ with $\alpha \delta-\beta \gamma \neq 0$ and $\psi(t)=1 / t^{2}$. Define $\mathscr{F}_{3}=\mathscr{B}_{3,2}$.

Theorem 2. Suppose that $\phi(t) \in \mathbb{Q}(t)$ has degree $d \geqq 2$. Let $x_{0} \in \mathbb{Q}$ and define $x_{n+1}=\phi\left(x_{n}\right)$ for each $n \geqq 0$, and suppose that the sequence $\left\{x_{n}\right\}_{n \geqq 0}$ is not eventually periodic. For any given $M \geqq 1$, the numerator of $x_{n+\Delta}-x_{n}$ has a doubly primitive prime factor for all $n \geqq 0$ and $M \geqq \Delta \geqq 1$, except for those pairs with $\Delta=1,2$ or 3 when $\phi \in \mathscr{F}_{1}, \mathscr{F}_{2}$ or $\mathscr{F}_{3}$, respectively, as well as for finitely many other exceptional pairs $(\Delta, n)$.

Proof of Theorem 2. We proceed by induction on $M$, the case $M=1$ being a consequence of Theorem 1. Suppose now that the result holds for all $M \leqq M^{\prime}-1$, and let us prove it holds for $M=M^{\prime} \geqq 2$. By the induction hypothesis, we find that the numerator of $x_{n+\Delta}-x_{n}$ has a doubly primitive prime factor $p_{\Delta, n}$ for $n \geqq 0$ and $M^{\prime}-1 \geqq \Delta \geqq 1$ other than for finitely many pairs $(\Delta, n)$, excluding those with $\Delta=1,2$ or 3 and $\phi \in \mathscr{F}_{1}, F_{2}$ or $F_{3}$, respectively. Set $\Delta=M^{\prime}$.

Let us suppose that $\phi$ does not belong to one of the corresponding exceptional classes of Lemma 5, in which case we can choose $\alpha \in \mathbb{P}^{1}(\overline{\mathbb{Q}})$ so that $\phi(\alpha)$ has exact period $\Delta$, $\phi(\alpha) \neq \infty$, and $\phi^{(\Delta)}(\alpha) \neq \alpha$. Proceeding as in the proof of Theorems $1^{\prime}$ and 1 , we obtain a sequence of prime ideals $\left\{P_{\Delta, n}\right\}$ of the Galois closure of $\mathbb{Q}(\alpha)$ such that $P_{\Delta, n}$ is a primitive prime factor of the numerator of $x_{n}-\alpha$ for all sufficiently large $n$. Moreover, if $p_{\Delta, n}$ is the 
rational prime divisible by $P_{\Delta, n}$, then $p_{\Delta, n}$ is a doubly primitive prime factor of the numerator of $x_{n+1+\Delta}-x_{n+1}$ by Lemma 6 , provided that $P_{\Delta, n}$ is not one of the finitely many prime ideal divisors of the numerator of $\phi^{(\Delta)}(\alpha)-\alpha$ or $\phi^{(l)}(\phi(\alpha))-\phi(\alpha)$ for some $1 \leqq l \leqq \Delta-1$, or of the denominator of $\alpha$ or $\phi(\alpha)$.

Now suppose that $\phi$ belongs to case (2) of Lemma 5, so that $\Delta=M^{\prime}=2$ and that $\phi(t)=\alpha+1 / g(t-\alpha)$ for some $\alpha \in \overline{\mathbb{Q}}$ and some quadratic polynomial $g(t)=b t^{2}+c t$ with $b c \neq 0$. Define $\psi(t)=\phi(t+\alpha)-\alpha=1 / g(t)$, so that 0 is periodic of period 2 for $\psi$ and $\psi(0)=\infty$. Note $\psi \notin \mathscr{T}$ (as in $\S 3$ ) since $g(t) \neq c t^{2}$. Define $y_{0}=x_{0}-\alpha$ and $y_{n+1}=\psi\left(y_{n}\right)$. Then $y_{n}=x_{n}-\alpha$ by induction. Let $K / \mathbb{Q}$ be a Galois extension containing $\mathbb{Q}(\alpha)$. Invoking Proposition $1^{\prime}$, we see there exists a prime ideal $P_{2, n}$ of $K$ that is a primitive prime factor of the numerator of $y_{n}=x_{n}-\alpha$ for all sufficiently large $n$. We exclude the finitely many prime ideals $P_{2, n}$ that divide the numerator or denominator of $b$ or $c$. Note that, since $\psi(x / y)=y^{2} / x(b x+c y), P_{2, n}$ is a primitive prime factor of the denominator of $y_{n-1}$, and hence for $N \geqq n-1$, we have that $P_{2, n}$ divides the numerator of $y_{N}$ if $N-n$ is even, and the denominator of $y_{N}$ if $N-n$ is odd. Moreover, if $e$ is the exact power of $P_{2, n}$ dividing the denominator of $y_{n-1}$, then $2^{l} e$ is the exact power of $P_{2, n}$ dividing the numerator of $y_{n+2 l-2}$ and the denominator of $y_{n+2 l-1}$ for all $l \geqq 1$. Hence if $a>b \geqq n-1$ then $P_{2, n}$ divides the numerator of $x_{a}-x_{b}=y_{a}-y_{b}$ if and only if $a \equiv b \equiv n(\bmod 2)$. Now suppose that $P_{2, n}$ divides the numerator of $x_{a}-x_{b}=y_{a}-y_{b}$ with $b<n-1$. If $a \leqq n-1$ we have $y_{n-1}=\psi^{(n-1-a)}\left(y_{a}\right) \equiv \psi^{(n-1-a)}\left(y_{b}\right)=y_{n-1-(a-b)}\left(\bmod P_{2, n}\right)$, so we may assume, without loss of generality that $a \geqq n-1$. Therefore $P_{2, n}$ divides the numerator or denominator of $y_{a}$, and so of $y_{b}$ (as $P_{2, n}$ divides the numerator of their difference), which contradicts primitivity. In summary, we have shown that the numerator of $x_{N+D}-x_{N}$ is divisible by $P_{2, n}$ if and only if $N \geqq n$ and $D \geqq 2$ is even, and since $x_{N+D}-x_{N}$ is rational, the same statement is true when $P_{2, n}$ is replaced by the rational prime $p_{2, n}$ dividing $P_{2, n}$. This completes the proof for the maps from case (2) of Lemma 5.

Now suppose that $\phi$ belongs to case (3) of Lemma 5, so that $\Delta=M^{\prime}=2$ and there exists a fractional linear tranformation $\sigma(t)=(\alpha t+\beta) /(\gamma t+\delta)$ such that $\phi=\sigma^{-1} \circ \psi \circ \sigma$ with $\psi(t)=1 / t^{2}$. Set $K=\mathbb{Q}(\alpha, \beta, \gamma, \delta)$, let $R$ be the ring of integers of $K$, and let $S$ be a finite set of prime ideals of $R$ such that the ring of $S$-integers $R_{S}$ is a principal ideal domain, and such that $\alpha, \beta, \gamma, \delta \in R_{S}$. (In particular, $S$ contains all prime ideals dividing the denominators of $\alpha, \beta, \gamma, \delta$.) Let $y_{0}=\sigma\left(x_{0}\right)$ and $y_{n+1}=\psi\left(y_{n}\right)$ for all $n \geqq 0$. For each $n$ choose $u_{n}, v_{n} \in R_{S}$ such that $y_{n}=u_{n} / v_{n}$ and $\left(u_{n}, v_{n}\right)=1$. Note that $y_{n}=y_{0}^{(-2)^{n}}=\sigma\left(x_{n}\right)$ for all $n \geqq 0$. Then

$$
x_{n}-x_{m}=\frac{(\alpha \delta-\beta \gamma)\left(y_{n}-y_{m}\right)}{\left(\gamma y_{m}-\alpha\right)\left(\gamma y_{n}-\alpha\right)}=\frac{(\alpha \delta-\beta \gamma)\left(u_{n} v_{m}-u_{m} v_{n}\right)}{\left(\gamma u_{m}-\alpha v_{m}\right)\left(\gamma u_{n}-\alpha v_{n}\right)}
$$

and in particular

$$
x_{n+1}-x_{n}=\frac{(\alpha \delta-\beta \gamma)\left(v_{n}^{2} v_{n}-u_{n} u_{n}^{2}\right)}{\left(\gamma u_{n}-\alpha v_{n}\right)\left(\gamma v_{n}^{2}-\alpha u_{n}^{2}\right)}=\frac{(\alpha \delta-\beta \gamma)\left(v_{n}^{3}-u_{n}^{3}\right)}{\left(\gamma u_{n}-\alpha v_{n}\right)\left(\gamma v_{n}^{2}-\alpha u_{n}^{2}\right)}
$$

and

$$
x_{n+2}-x_{n}=\frac{(\alpha \delta-\beta \gamma)\left(u_{n}^{4} v_{n}-u_{n} v_{n}^{4}\right)}{\left(\gamma u_{n}-\alpha v_{n}\right)\left(\gamma u_{n}^{4}-\alpha v_{n}^{4}\right)}=-\frac{(\alpha \delta-\beta \gamma) u_{n} v_{n}\left(v_{n}^{3}-u_{n}^{3}\right)}{\left(\gamma u_{n}-\alpha v_{n}\right)\left(\gamma u_{n}^{4}-\alpha v_{n}^{4}\right)} .
$$


Let $\eta=\gamma u_{n}-\alpha v_{n}$. Now $\left(u_{n}, v_{n}\right)=(1)$ so that $\left(v_{n}^{3}-u_{n}^{3}, u_{n}\right)=\left(v_{n}^{3}-u_{n}^{3}, v_{n}\right)=(1)$, an equality of $R_{S}$-ideals. Hence

$$
\begin{aligned}
\left(v_{n}^{3}-u_{n}^{3}, \eta\left(\gamma v_{n}^{2}-\alpha u_{n}^{2}\right)\right) & =\left(v_{n}^{3}-u_{n}^{3}, \eta\left(\gamma v_{n}^{3}-\alpha u_{n}^{2} v_{n}\right)\right)=\left(v_{n}^{3}-u_{n}^{3}, \eta\left(\gamma u_{n}^{3}-\alpha u_{n}^{2} v_{n}\right)\right) \\
& =\left(v_{n}^{3}-u_{n}^{3}, \eta^{2}\right) .
\end{aligned}
$$

Similarly $\left(v_{n}^{3}-u_{n}^{3}, \eta\left(\gamma u_{n}^{4}-\alpha v_{n}^{4}\right)\right)=\left(v_{n}^{3}-u_{n}^{3}, \eta^{2}\right)$. Hence the prime factors in the numerator of $x_{n+2}-x_{n}$ are a subset of those in the numerator of $x_{n+1}-x_{n}$, and those dividing $u_{n} v_{n}$, which are the same as those dividing $u_{0} v_{0}$. We deduce that there can be no doubly primitive prime factor of the numerator of $x_{n+2}-x_{n}$ for any $n \geqq 2$. This completes the proof for the maps from case (3) of Lemma 5.

Finally, if $\Delta=2$ or 3 and $\phi \in \mathscr{B}_{\Delta, d}$, that is cases (4) and (5) of Lemma 5, then Corollary 2 of $\S 7$ shows $x_{n+\Delta}-x_{n}$ fails to have a doubly primitive prime factor in its numerator when $n$ is large.

\section{The density of prime divisors of dynamical sequences}

Given a sequence $\left\{x_{n}\right\}_{n \geq 0}$, let $\mathscr{P}$ be the set of primes which divide the numerator of some non-zero element $x_{n}$, and $\mathscr{P}(x)$ be the number of elements of $\mathscr{P}$ up to $x$. We will prove that for the Fermat numbers, one has $\mathscr{P}(x) \ll x^{1 / 2} / \log x$. Although this bound is small compared to the total number of primes up to $x$, a simple heuristic indicates that the true order of magnitude of $\mathscr{P}(x)$ is probably some power of $\log \log x$ !

Select integers $m$ and $N$ so that $2^{m} \approx x^{1 / 2}$ and $2^{N} \approx x^{2 / 3}$. There are $\ll 2^{m} / m$ prime factors of $F_{0} F_{1} \cdots F_{m-1}=2^{2^{m}}-1$ by the prime number theorem. Any prime divisor $p_{n}$ of $F_{n}=2^{2^{n}}+1$ is $\equiv 1\left(\bmod 2^{n+1}\right)$. There are $\leqq x / 2^{n}$ integers in this arithmetic progression, and so $\ll x / 2^{N}$ such primes, in total, with $n \geqq N$. The Brun/Titchmarsh Theorem tells us that there are $\ll x / 2^{n} \log \left(x / 2^{n}\right)$ primes $\equiv 1\left(\bmod 2^{n+1}\right)$ up to $x$. If $m \leqq n<N$, this is $\ll x / 2^{n} \log x$, and so there are $\ll x / 2^{m} \log x$ such primes in total. Combining these observations yields the claim that $\mathscr{P}(x) \ll x^{1 / 2} / \log x$.

Presumably if $\left\{x_{n}\right\}_{n \geq 0}$ is a dynamical sequence, obtained from a rational function of degree $d \geqq 2$, then it might be possible to prove something like $\mathscr{P}(x) \ll x^{1-1 / d+o(1)}$ (except for certain degenerate cases, such as $x_{n}$ defined as iterates of $\phi(t)=(t+p)^{2}-p$ for any prime $p$ ). We expect that the prime divisors of $x_{n}$ belong to an increasingly sparse sequence as $n$ gets larger, since the $x_{n}$ are values of the iterated function $\phi^{(m)}(t)$ for all $n \geqq m$. Some result of this type should be accessible from a study of the Galois groups of these extensions. In fact there are several interesting results in the literature. First, Odoni [9] showed that for the Euclid numbers $E_{n}$ (where $E_{0}=2$ and $E_{n+1}=E_{n}^{2}-E_{n}+1$ ) we have $\mathscr{P}(x) \ll \pi(x) / \log \log \log x$; and then in [10], the remarkable result that for "almost all" monic $\phi(t) \in \mathbb{Z}[t]$ of given degree $\geqq 2$ and given height, $\mathscr{P}(x)=o(\pi(x))$ no matter what the value of $x_{0} \in \mathbb{Z}$. Recently Jones [6] showed that $\mathscr{P}(x)=o(\pi(x))$, no matter what the value of $x_{0} \in \mathbb{Z}$, for the polynomials $\phi(t)=t(t-a)+a, t^{2}+a t-1(a \neq 0,2)$, $t^{2}+a(a \neq-1), t^{2}-2 a t+a(a \neq-1,1)$, where $a \in \mathbb{Z}$. 


\section{Appendix A. On complex dynamics}

Here we collect a few results that lie in the realm of complex dynamics on $\mathbb{P}^{1}(\mathbb{C})$ viewed as a Riemann surface. They are all well-known, and we either point the reader to a proof or give our own if it is brief.

Let $\phi(t) \in \mathbb{C}(t)$ be a rational function and let $P \in \mathbb{P}^{1}(\mathbb{C})$ be a fixed point of $\phi$. The fixed point multiplicity of $P \in \mathbb{C}$ is the order of vanishing of $\phi(t)-t$ at $t=P$. If $P=\infty$, we observe that $1 / \phi(1 / t)$ has a fixed point at $t=0$, and we define the fixed point multiplicity of $P=\infty$ to be the order of vanishing of $1 / \phi(1 / t)-t$ at the origin. Note that $1 / \phi(1 / t)=\left(\sigma^{-1} \circ \phi \circ \sigma\right)(t)$, where $\sigma(t)=1 / t$ is a fractional linear change of coordinates.

Continuing with the notation from the last paragraph, we define the fixed point multiplier $\lambda_{P}$ to be the derivative $\phi^{\prime}(P)$ if $P \neq \infty$. If $P=\infty$, define the fixed point multiplier to be

$$
\lambda_{\infty}=\left.\frac{d}{d t}\left(\frac{1}{\phi(1 / t)}\right)\right|_{t=0}=\left.\frac{d}{d t}\left(\sigma^{-1} \circ \phi \circ \sigma\right)(t)\right|_{t=0},
$$

where as above, $\sigma(t)=1 / t$.

Next let us suppose that $P \in \mathbb{P}^{1}(\mathbb{C})$ is a periodic point of (exact) period $m$; i.e., $\phi^{(m)}(P)=P$ and this relation is false if we replace $m$ by any smaller positive integer. Then $\phi^{(n)}(P)=P$ for any integer $n$ divisible by $m$ since

$$
\phi^{(n)}(P)=\left(\phi^{(m)} \circ \phi^{(m)} \circ \cdots \circ \phi^{(m)}\right)(P)=P .
$$

Conversely, if $\phi^{(n)}(P)=P$, then $m \mid n$, for if we write $n=m q+r$ for some $0 \leqq r<m$, then $\phi^{(r)}(P)=\phi^{(r)}\left(\phi^{(m q)}(P)\right)=\phi^{(n)}(P)=P$, which implies $r=0$ by minimality.

Lemma A.1. Let $\phi(t)$ be a rational function of degree $d \geqq 2$ and let $P \in \mathbb{P}^{1}(\mathbb{C})$ be a fixed point of $\phi(t)$. The fixed point multiplicity of $P$ is greater than one if and only if $\lambda_{P}=1$. In particular, if $\lambda_{P}=0$, then the fixed point multiplicity of $P$ is exactly 1.

Proof. We may assume that $P \neq \infty$ by replacing $\phi(t)$ with $1 / \phi(1 / t)$ and replacing $P=\infty$ with $P=0$ if necessary. Note that by definition this does not affect the fixed point multiplicity or the multiplier. Moreover, we may replace $\phi(t)$ by $\phi(t+P)-P$ in order to assume that $P=0$. By the chain rule, this does not affect the fixed point multiplier. The fixed point multiplicity is unaffected because $\phi(t+P)-P-t=\phi(t+P)-(t+P)$ has a zero of order $m$ at $t=0$ if and only if $\phi(t)-t$ has a zero of order $m$ at $t=P$.

To prove the first assertion, we expand as a power series about the origin: $\phi(t)=\lambda_{0} t+c_{2} t^{2}+c_{3} t^{3}+\cdots$. Then $\phi(t)-t=\left(\lambda_{0}-1\right) t+c_{2} t^{2}+\cdots$ and the result follows.

Lemma A.2. A rational function $\phi(t)$ of degree $d \geqq 2$ has exactly $d+1$ fixed points when counted with multiplicity.

Proof. Choose a fractional linear change of coordinates $\sigma(t)=(\alpha t+\beta) /(\gamma t+\delta)$ with $\alpha \delta-\beta \gamma \neq 0$, and consider the new rational function $\left(\sigma^{-1} \circ \phi \circ \sigma\right)(t)$. One can choose $\sigma$ so that any three given distinct points of $\mathbb{P}^{1}(\mathbb{C})$ are sent to any other three given distinct points; so, for example, we may assume that $\infty$ is not a fixed point. (As $d \geqq 2$ there must 
exist at least one non-fixed point, say because $\phi(t)-t$ is not the zero function.) Changing coordinates doesn't change the derivatives of $\phi(t)$ at a fixed point (by the chain rule), and hence does not affect the multiplicity of a fixed point. Now $\phi(t)=f(t) / g(t)$ where $\operatorname{deg} f \leqq \operatorname{deg} g=d$. The fixed points are exactly the solutions to the equation $\phi(t)=t$, or equivalently, the roots of $f(t)=\operatorname{tg}(t)$. This equation has degree $d+1$, and hence exactly $d+1$ roots when counted with multiplicity.

Lemma A.3. Let $\phi(t)$ be a rational function of degree $d \geqq 2$ and let $P \in \mathbb{P}^{1}(\mathbb{C})$ be a fixed point of $\phi(t)$, in which case $P$ is also a fixed point of $\phi^{(2)}(t)$.

(1) If the fixed point multiplicity of $P$ for $\phi$ is $\ell>1$, then the fixed point multiplicity of $P$ for $\phi^{(2)}$ is $\ell$.

(2) Suppose the fixed point multiplicity of $P$ is 1 for $\phi$ and $\ell>1$ for $\phi^{(2)}$. Then $\ell=2 k+1$ for some positive integer $k$. Moreover, there exist $k$ distinct critical points $Q_{1}, \ldots, Q_{k}$ for $\phi$-i.e., $Q_{i} \in \mathbb{P}^{1}(\mathbb{C})$ with $\phi^{\prime}\left(Q_{i}\right)=0$ - such that $\phi^{(m)}\left(Q_{i}\right) \rightarrow P$ as $m \rightarrow \infty$.

Proof. We may assume that $P \neq \infty$ by replacing $\phi(t)$ with $1 / \phi(1 / t)$ and replacing $P=\infty$ with $P=0$ if necessary. Note that by definition this does not affect the fixed point multiplicities or multipliers. To prove (1), we expand as a power series about the origin: $\phi(t)-t=c_{\ell} t^{\ell}+\cdots$, or $\phi(t)=t+c_{\ell} t^{\ell}+\cdots$. Iterating shows $\phi^{(2)}(t)-t=2 c_{\ell} t^{\ell}+\cdots$, from which (1) follows.

The proof of (2) is significantly more difficult. See [1], Lemma 4, or [8], Lemmas 10.4 and 10.11 .

\section{Appendix B. Kisaka's classification of exceptions to Baker's Theorem}

Two rational maps $\phi(t), \psi(t) \in \mathbb{C}(t)$ are said to be conjugate if $\phi=\sigma^{-1} \circ \psi \circ \sigma$ for some fractional linear transformation $\sigma(t)=(\alpha t+\beta) /(\gamma t+\delta)$ with $\alpha \delta-\beta \gamma \neq 0$.

In [7], Theorem 1, Kisaka showed that if $\phi(t) \in \mathbb{C}(t)$ is a rational map of degree $d \geqq 2$ with no periodic point of exact period $\Delta$, then $\phi$ is conjugate to one of the following:

(1) $(\Delta, d)=(2,2)$ :

$$
\psi(t)=\frac{t^{2}-t}{a t+1} \quad(a \neq-1)
$$

(2) $(\Delta, d)=(2,3)$ :

$$
\begin{array}{ll}
\psi(t)=\frac{t^{3}+a t^{2}-t}{\left(a^{2}-1\right) t^{2}-2 a t+1} & (a \neq 0) ; \text { or } \\
\psi(t)=\frac{t^{3}-t}{-t^{2}+b t+1} & (b \neq 0) ; \text { or } \\
\psi(t)=\frac{t^{3}+\frac{4}{c} t^{2}-t}{-t^{2}+c t+1} & (c \neq 0, \pm 2 i) .
\end{array}
$$


(3) $(\Delta, d)=(2,4)$ :

$$
\begin{gathered}
\psi(t)=\frac{t^{4}-t}{-2 t^{3}+1} \text { or } \frac{t^{4}+t^{3}+t^{2}-t}{-t^{3}+t^{2}-3 t+1} \text { or } \frac{t^{4}-3^{\frac{1}{3}} \cdot t^{3}+3^{\frac{2}{3}} \cdot t^{2}-t}{-t^{3}+3^{\frac{4}{3}} \cdot t^{2}-5 \cdot 3^{-\frac{1}{3}} \cdot t+1} \\
\quad \text { or } \frac{t^{4}+\bar{c}_{0} t^{3}+\bar{b}_{0} t^{2}-t}{-t^{3}+b_{0} t^{2}+c_{0} t+1}
\end{gathered}
$$

where $\left(x-b_{0}\right)\left(x-\bar{b}_{0}\right)=x^{2}-3 x+1$ and $\left(x-c_{0}\right)\left(x-\bar{c}_{0}\right)=x^{2}+5 x+5$.

(4) $(\Delta, d)=(3,2)$ :

$$
\psi(t)=\frac{t^{2}+\omega t}{\frac{\omega+5}{\omega-1} t+1} \text { or } \frac{t^{2}+\omega t}{\omega t+1}
$$

where $\omega$ is a primitive third root of unity.

Note added in proof. A collision between the terminologies for discussing ramification in algebraic number theory and in complex dynamics has led to the following nonstandard usage in Lemmas 2, 3, and 5: We say that $\phi$ is totally ramified at $y$ if $\phi^{-1}(y)$ is a single point.

Erratum added in proof. In Lemma 5, there should be an extra exceptional case $\left(2^{\prime}\right)$ : $\Delta=2$ and $\phi=\sigma^{-1} \circ \psi \circ \sigma$ for some $\sigma(t)=\lambda t+\beta$ with $\lambda \neq 0$, and $\psi(t)=1 /\left(t^{3}+2 t^{2}+2 t\right)$. It arises because the final two displayed expressions in the proof of the lemma may share a non-obvious common factor for certain choices of $b$. By taking an appropriate resultant, one sees that $b= \pm 2, \pm 2 i$ are precisely the values that yield such a common factor. Taking $c$ as in the proof, this gives $\phi(t)=1 /\left(t^{3}+2 i^{m} t^{2}+2(-1)^{m} t\right)$. Replacing $\phi$ with $i^{-m} \phi\left(i^{m} t\right)$ gives $\psi$.

The statement of Theorem 2 is unaffected by this new exception to Lemma 5. Indeed, the strategy of the third paragraph of the proof carries over mutatis mutandis to this case.

\section{References}

[1] I. N. Baker, Fixpoints of polynomials and rational functions, J. London Math. Soc. 39 (1964), 615-622.

[2] E. Bombieri and W. Gubler, Heights in diophantine geometry, New Math. Monogr. 4, Cambridge Univ. Press, Cambridge 2006.

[3] R. D. Carmichael, On the numerical factors of arithmetical forms $\alpha^{n} \pm \beta^{n}$, Ann. Math. (2) 15 (1913), 30-70.

[4] M. Hindry and J. H. Silverman, Diophantine geometry: An introduction, Springer Verlag, New York 2000.

[5] P. Ingram and J. H. Silverman, Primitive divisors in arithmetic dynamics, Math. Proc. Cambridge Philos. Soc. 146 (2009), 289-302.

[6] R. Jones, The density of prime divisors in the arithmetic dynamics of quadratic polynomials, J. Lond. Math. Soc. 78 (2008), 523-544.

[7] M. Kisaka, On some exceptional rational maps, Proc. Japan Acad. (A) 71 (1995), 35-38.

[8] J. Milnor, Dynamics in one complex variable, Introductory Lectures, 2nd ed., Friedr. Vieweg \& Sohn, Braunschweig 2000.

[9] R.W. K. Odoni, On the prime divisors of the sequence $w_{n+1}=1+w_{1} \cdots w_{n}$, J. Lond. Math. Soc. 32 (1985), 1-11. 
[10] R. W. K. Odoni, The Galois theory of iterates and composites of polynomials, Proc. Lond. Math. Soc. 51 (1985), 385-414.

[11] B. Rice, Primitive prime divisors in polynomial arithmetic dynamics, Integers 7 (2007), A26.

[12] J. H. Silverman, The arithmetic of dynamical systems, Springer-Verlag, New York 2007.

\section{McGill University, Montréal QC H3A 2K6, Canada} e-mail: xander@math.mcgill.ca

Université de Montréal, Montréal QC H3C 3J7, Canada e-mail: andrew@DMS.UMontreal.CA

Eingegangen 21. Oktober 2009, in revidierter Fassung 18. Juli 2010 